See discussions, stats, and author profiles for this publication at: https://www.researchgate.net/publication/260228788

\title{
Preference Reversals: Time and Again
}

Article in Journal of Risk and Uncertainty · February 2016

DOI: 10.1007/s11166-016-9233-z

\section{CITATIONS}

5

4 authors, including:

8

Carlos Alós-Ferrer

University of Zurich

106 PUBLICATIONS 1,103 CITATIONS

SEE PROFILE

Alexander K. Wagner

University of Vienna

14 PUBLICATIONS 98 CITATIONS

SEE PROFILE
(2.) Georg D. Granic

14 PUBLICATIONS 38 CITATIONS

SEE PROFILE 


\title{
Preference Reversals: Time and Again*
}

\author{
Carlos Alós-Ferrer ${ }^{a} \quad$ Dura-Georg Granić ${ }^{b} \quad$ Johannes Kern $^{c}$ \\ Alexander K. Wagner ${ }^{d}$
}

August, 2015

Forthcoming, Journal of Risk and Uncertainty

\begin{abstract}
This paper sheds new light on the preference reversal phenomenon by analyzing decision times in the choice task. In a first experiment, we replicated the standard reversal pattern and found that choices associated with reversals take significantly longer than non-reversals, and non-reversal choices take longer whenever long-shot lotteries are selected. These results can be explained by a combination of noisy lottery evaluations (imprecise preferences) and an overpricing phenomenon associated with the compatibility hypothesis. The first cause explains the existence of reversals, while the second explains the predominance of a particular type thereof. A second experiment showed that the overpricing phenomenon can be shut down, greatly reducing reversals, by using ranking-based, ordinally-framed evaluation tasks. This experiment also disentangled the two determinants of reversals, because imprecise evaluations still deliver testable predictions on decision times even in the absence of the overpricing phenomenon. Strikingly, when unframed ranking tasks were used, decision times in the choice phase were greatly reduced, even though this phase was identical across treatments. This observation is consistent with psychological insights on conflicting decision processes.
\end{abstract}

Keywords: Preference reversals, Decision times, Imprecise preferences, Compatibility hypothesis

${ }^{*}$ The authors gratefully acknowledge helpful comments from an anonymous referee, Mónica Capra, Urs Fischbacher, Nikos Georgantzis, Werner Güth, and seminar participants at Ca' Foscari University (Venice), Emory University (Atlanta), University of Indiana at Bloomington, University of Innsbruck, Universidad Jaume I (Castellón), the TIBER XI conference in Tilburg, the Economic Science Association 2012 conference in Cologne, and the FUR XVI conference in Rotterdam.

${ }^{a}$ Department of Economics, University of Cologne, Albertus-Magnus Platz, D-50923 Cologne, Germany. Email: carlos.alos-ferrer@uni-koeln.de

${ }^{b}$ Department of Economics, University of Cologne, Albertus-Magnus Platz, D-50923 Cologne, Germany. Email: georg.granic@uni-koeln.de

${ }^{c}$ Department of Economics, University of Cologne, Albertus-Magnus Platz, D-50923 Cologne, Germany. Email: johannes.kern@uni-koeln.de

${ }^{d}$ Department of Economics, University of Cologne, Albertus-Magnus Platz, D-50923 Cologne, Germany. Present Address: Vienna Center for Experimental Economics and Department of Economics, University of Vienna, Oskar-Morgenstern-Platz 1, A-1090 Vienna, Austria. Email: alexander.k.wagner@univie.ac.at 


\section{Introduction}

The concept of preference is of fundamental importance for decision theory and economic analysis. Yet, preferences are not a primitive but a derived object which structures choices as long as they exhibit some basic consistency, e.g. in the form of the weak axiom of revealed preference. If choices are consistent, a number of elementary predictions can be derived, which form the basis for decision theory, microeconomics, consumer research, and judgment and decision making. One such prediction is that choices should agree with valuations: if a decision maker chooses one option over another, he should value the former more than the latter.

This common-sense prediction is at odds with observed decisions under risk. The preference reversal phenomenon, first documented in psychology by Slovic and Lichtenstein (1968) and Lindman (1971), describes a situation in which participants are asked to state monetary valuations for a series of lotteries (usually through minimum selling prices), and separately choose from pairs of those lotteries. The pairs consist of a $P$-bet, which has a high probability of paying a moderate amount of money, and a $\$$-bet, which has a low probability of paying a high amount of money. A preference reversal occurs if either the $P$-bet is chosen from a pair in which the $\$$-bet is priced higher or the $\$$-bet is chosen from a pair in which the $P$-bet is priced higher. The preference reversal phenomenon is characterized by a high rate of reversals of the first type (between 40 and 80 percent in most experiments), which are called predicted reversals. Reversals of the second type, termed unpredicted, are less frequent (between 5 and 30 percent). The asymmetry between both types of reversals is especially problematic, for, if reversals were due to e.g. participants' errors, one should expect similar numbers of both types. In other words, while one could explain away unpredicted reversals as noisy observations, predicted reversals remain a serious challenge to basic economic analysis.

It is no surprise that preference reversals have received a great deal of attention in the last half century. After the first replication in economics by Grether and Plott (1979), a large number of experimental and theoretical studies has shown that the phenomenon is extremely stable. It has been replicated using hypothetical and real payments, different payment schemes, and different elicitation methods for lottery prices (for a survey, see e.g. Seidl, 2002). Preference reversals of this particular form have been documented beyond lottery choice, e.g. in the field of health utility measurements (Stalmeier et al., 1997; Bleichrodt and Pinto Prades, 1994; Oliver, 2013). Furthermore, other forms of inconsistencies between different preference elicitation methods have been established in the literature, including reversals between pricing and rating (Schkade and Johnson, 1989) as well as discrepancies between certainty and probability equivalents (Hershey and Schoemaker, 1985; Johnson and Schkade, 1989; Delquié, 1993). In addition to their conceptual importance for decision theory, these phenomena are of great relevance for applied economics, since they cast doubts on the validity of e.g. consumer valuations, and, accordingly, on demand estimations and policy decisions based on those valuations.

The present research provides new evidence on the determinants of preference reversals by investigating decision times in two separate experiments. This allows us to go beyond existing accounts of behavioral data and investigate the decision processes underlying reversals. Using a well-established, stylized fact on decision times (that decisions take longer when the decision 
maker is closer to indifference), we argue that the combination of two standard elements of existing accounts of preference reversals is enough to explain the pattern of reversals while delivering testable predictions on decision times. The two determinants are the imprecision of preferences in the evaluation phase (Schmidt and Hey, 2004; Butler and Loomes, 2007) and the overpricing of $\$$-bets. The latter can be due to anchoring of evaluations on the largest monetary outcomes of a lottery (Tversky et al., 1990), which is itself a consequence of the cardinal/monetary framing of the evaluation phase. An alternative explanation for the overpricing of $\$$-bets is reference dependence (Sugden, 2003; Schmidt et al., 2008; Lindsay, 2013). ${ }^{1}$

The main, clear-cut prediction that we obtain for decision times is that choices associated with reversals of either type are slower than the corresponding non-reversal choices. Our first experiment reproduced the standard preference reversal pattern and confirmed this prediction. We employed two different payment methods, the BDM procedure (Becker et al., 1964) and an ordinal payment scheme (Goldstein and Einhorn, 1987; Tversky et al., 1990; Cubitt et al., 2004), showing that our findings are robust to changes in the evaluation task.

Our first experiment hence provided novel evidence in favor of theories based on preference imprecision and the overpricing of $\$$-bets. In our second experiment, we showed that these two determinants of preference reversals can be disentangled in the laboratory by "shutting down" the overpricing phenomenon. This was accomplished by moving away from cardinal elicitation tasks. Instead, we employed two different ranking methods (plus a control BDM replication), one with a price framing, and one where we carefully removed all references to prices. This experiment helps establish that the imprecision of elicited monetary valuations is an important key for the existence of reversals, while the overpricing phenomenon causes their asymmetry, i.e. the predominance of predicted preference reversals. When the latter is eliminated, predicted reversals become rare, but choices associated with reversals remain significantly slower than corresponding non-reversal choices. This confirms our interpretation because this prediction can be shown to arise exclusively from preference imprecision in the choice phase.

Received evidence on preference reversals could potentially be explained by a number of alternative, "as if" models. The analysis of decision times, however, allows us to put different hypotheses on the determinants of preference reversals to a more stringent test than if we relied on behavioral data only, and further enables us (through predictions arising from one determinant only) to disentangle the causes of reversals. ${ }^{2}$

Our research also delivers additional theoretical and methodological insights. On the basis of our assumptions, we also obtain an additional, surprising prediction, namely that decisions where the riskier $\$$-bet is chosen without giving rise to a reversal should be slower than those non-reversals where the $P$-bet is chosen. This nontrivial prediction arises as a consequence

\footnotetext{
1 Reference dependence states that a subject's reference point when asked for a minimum selling price is the lottery at hand. Exchanging the lottery for a certain amount of cash involves a probabilistic loss which is higher for the $\$$-bet than for the $P$-bet leading to a higher stated price for the $\$$-bet. We thank an anonymous referee for referring us to this strand of literature.

2 The measurement of decision times is a standard tool in psychology (see, e.g., Bargh and Chartrand, 2000). To our knowledge, the first studies in economics employing them to study risky choice were those of Wilcox $(1993,1994)$. Decision times were also used by Moffatt (2005) relying on risky-choice data from Hey (2001). Rubinstein (2007) advocated the measurement of decision times in large-scale, web-based experiments to better understand economic decisions. Achtziger and Alós-Ferrer (2014) measured response times within a Bayesian-updating paradigm in order to study intuitive decision making in economic contexts.
} 
of the conjunction of imprecise preferences and the overpricing phenomenon, and hence was predicted for (and observed in) the first experiment but not for the second. A further, striking observation was that choices in the treatment with unframed ranking-based evaluations were much faster than those in other treatments, in spite of the fact that choice phases were identical across treatments. This fact has a simple process-based explanation (which we relegate to the discussion of that experiment). Last, our design specifically allowed comparing reversal rates when prices are elicited before the choice phase to reversal rates occurring when prices are elicited after the choice phase. This comparison, which has not been previously made in the literature, was motivated by evidence from psychology (see Section 2.3 below) indicating that choices might sharpen and even modify previously imprecise preferences. In agreement with this observation, we show that ordering effects, although small, are present in the measurement of reversals.

The remainder of the paper is organized as follows. In Section 2 we derive our experimental hypotheses from a simple formal model. Sections 3 and 4 describe the first and second experiments and their results, respectively. Section 5 concludes.

\section{A Simple Model of Preference Reversals and Decision Times}

In this section we derive our experimental hypotheses from a simplified formal model. We develop this model only to the extent necessary to obtain predictions on preference reversals and the associated decision times. In particular, the model is tailored to experiments on preference reversals and makes no attempt to provide a foundation based on first principles. Rather, we take the main building blocks directly in as assumptions, including imprecise monetary evaluations and the overpricing phenomenon.

\section{$2.1 \quad$ Model and Rationale}

We consider a choice between a $P$-bet and a $\$$-bet and the pricing decisions for both bets. In preference reversal experiments, results refer to a relatively large number of evaluation and choice decisions. Systematic biases are avoided, e.g. by offering choices between lotteries of similar expected values, or counterbalancing the difference in expected values across pairs. Hence, to obtain experimental hypotheses, it is reasonable to treat the utilities of the bets as random variables. Specifically, let $u_{P}$ and $u_{\$}$ denote the "true" utilities of the $P$-bet and the $\$$-bet, respectively. We assume that, in an experiment, these utilities are drawn from i.i.d. continuous random variables with some fixed distribution. However, the analysis relies only on the distribution of utility differences $u_{P}-u_{\$}$. We assume that this distribution has an everywhere positive density $h .^{3}$

The first building block of our model is preference imprecision in the evaluation phase. We follow Schmidt and Hey (2004) and Butler and Loomes (2007), who found that subjects in preference reversal experiments exhibit imprecise monetary valuations of lotteries. Specifically,

\footnotetext{
3 Since $u_{P}$ and $u_{\$}$ are i.i.d, $u_{P}-u_{\$}$ and $u_{\$}-u_{P}$ have the same distribution. If the distribution of $u_{P}$ and $u_{\$}$ has density $v$ then $h=\left(v * v^{-}\right)$, where $v^{-}(s)=v(-s)$ for all $s$ and the symbol $*$ denotes the convolution operator.
} 
Schmidt and Hey (2004) suggested that part of the preference reversal phenomenon might be due to pricing errors, while choice errors play a minor role. ${ }^{4}$ Our model incorporates these observations by assuming a noisy evaluation phase and a relatively noise-free choice phase. The first assumption states that the pricing of lotteries is a noisy process. Denote by $C E_{P}$ and $C E_{\$}$ the elicited certainty equivalents of the $P$-bet and the $\$$-bet, respectively.

Assumption 1. $C E_{P}=u_{P}+\zeta_{P}$ and $C E_{\$}=u_{\$}+\zeta_{\$}$, where $\zeta_{P}$ and $\zeta_{\$}$ are independent error terms with everywhere positive density functions. ${ }^{5}$

In contrast, the choice phase should be comparatively noise-free. For simplicity, the second assumption postulates that choices follow the underlying utilities. Write $c(P, \$)=P$ if the $P$-bet was chosen in the choice task and $c(P, \$)=\$$ if the $\$$-bet was chosen.

Assumption 2. $c(P, \$)=P$ whenever $u_{p}>u_{\$}$ and $c(P, \$)=\$$ whenever $u_{p}<u_{\$}$.

The second element of our model is the compatibility hypothesis proposed by Tversky et al. (1988, 1990), according to which attributes that naturally map onto the evaluation scale are given predominant weight in the evaluation phase. Since the evaluation scale usually refers to prices, the monetary outcomes of the lotteries might anchor valuations, giving rise to an overpricing of the $\$$-bet, where a large monetary outcome is salient. ${ }^{6}$ In other words, since the $\$$-bet yields a large outcome with moderate probability and the $P$-bet pays a moderate outcome with high probability, subjects will tend to state a higher price for the $\$$-bet. This overpricing phenomenon can be captured by simply assuming a strictly positive mean for the error term associated with the valuation of the $\$$-bet.

Assumption 3. There is a tendency to overprice the $\$$-bet, i.e. $E\left[\zeta_{\$}\right]=K>0$ but $E\left[\zeta_{P}\right]=0$. Further, the densities of $\zeta_{P}$ and $\zeta_{\$}$ are symmetric around the means and unimodal.

The last assumption refers to decision times. It is a well-established fact in the psychological literature that decision times reflect preferences in the sense that hard choices, where the decision maker is close to being indifferent, result in longer decision times than easy choices, where one option is clearly better (see e.g. Shultz et al., 1999; Sharot et al., 2009; Alós-Ferrer et al., 2012). This has also been observed in the risky-choice studies of Wilcox (1993) and Moffatt (2005), and in a study of intertemporal choices by Chabris et al. (2009). To model this effect in a simple way, we postulate that the choice time $D T_{C}$ only depends on the utility difference $\left|u_{P}-u_{\$}\right|$. To avoid unnecessarily complicating the model, we make the simplifying assumption that decisions are of two kinds, easy and hard. Easy decisions correspond to utility pairs $\left(u_{p}, u_{\$}\right)$ such that $\left|u_{P}-u_{\$}\right| \geq \delta$ for some $\delta>0$, while utility pairs $\left(u_{p}, u_{\S}\right)$ with $\left|u_{P}-u_{\$}\right|<\delta$ lead to hard decisions.

\footnotetext{
4 See Blavatsky (2009) for a formal model focused on those findings.

5 An alternative interpretation of $u_{P}$ and $u_{\$}$ is hence that they correspond to the expected monetary valuations of the lotteries, in the absence of (over)pricing biases. The second part of the assumption is for technical convenience. The analysis goes through, with more cumbersome proofs, if the error terms have bounded support.

6 Tversky et al. (1990) used a design with additional choices between the bets and cash amounts and showed that at least part of the predicted reversals arise because of an overpricing of \$-bets. Tversky et al. (1988) also proposed the prominence hypothesis, which assumes a bias in the choice stage rather than in the evaluation stage (see also Fischer et al., 1999). Cubitt et al. (2004) investigated a number of alternative hypotheses including prominence and compatibility and dismissed each of them in isolation, concluding that a combination of hypotheses would be a more reasonable explanation of their findings.
} 
Denote by $T_{E}=E\left[D T_{C}|| u_{P}-u_{\$} \mid \geq \delta\right]$ and $T_{H}=E\left[D T_{C}|| u_{P}-u_{\$} \mid<\delta\right]$ the expected choice times for easy and hard decisions, respectively. The next assumption captures the idea that choice decisions in which a subject is close to indifference between two items are harder than "obvious" choices.

Assumption 4. Hard choices take longer than easy choices, i.e. $T_{H}>T_{E}$.

Our assumptions are meant to reflect the basic principles involved in preference reversal experiments without unnecessarily complicating the exposition and the analysis. Of course, one could postulate more involved formulations, as e.g. a continuously monotonic relation between choice times and closeness to indifference. The next section shows that the simple versions postulated above are enough to provide testable hypotheses.

\subsection{Predictions}

Our model makes several predictions which can be experimentally tested (all proofs are in the online appendix). The first one is that predicted reversals should be more frequent than unpredicted ones, that is, we expect the usual pattern of preference reversals. This is unsurprising, since our model incorporates standard elements which have been developed precisely to explain this pattern. We view this result simply as a basic test that the model generates reasonable predictions.

Proposition 1. Under Assumptions 1, 2, and 3, the reversal rate is higher for predicted preference reversals than for unpredicted preference reversals, i.e. $\operatorname{Pr}\left(C E_{\$}>C E_{P} \mid c(P, \$)=P\right)>$ $\operatorname{Pr}\left(C E_{P}>C E_{\$} \mid c(P, \$)=\$\right)$.

The intuition for this result is straightforward. Both kinds of reversals result from noise in the evaluation phase shifting the evaluations of the lotteries in opposite directions. A reversal occurs when, due to noisy realizations, the evaluation ranking is reversed with respect to the one derived from utilities. The overpricing phenomenon helps produce predicted reversals: initially, the $\$$-bet is ranked lower than the $P$-bet $\left(u_{\$}<u_{P}\right)$, but overpricing tends to shift the valuation of the $\$$-bet higher than that of the $P$-bet. Overpricing, however, makes unpredicted reversals less likely: the $\$$-bet is initially ranked higher and overpricing tends to increase its evaluation with respect to the $P$-bet even more.

We can reformulate the prediction arising from the last proposition straight away as an experimental hypothesis.

H1 The average rate of predicted reversals (i.e. percentage of reversals over all $P$-choices) per subject is larger than the average rate of unpredicted reversals (i.e. percentage of reversals over all \$-choices).

The novel feature in our experimental design is that we record decision times in the choice task which allows us to look at preference reversals from a different perspective. Assumption 4 yields two novel predictions for decision times. The first refers to decision times for choices leading to preference reversals vs. choices not leading to preference reversals. 
Proposition 2. Let $D T_{C}$ denote the decision time in the choice phase. Under Assumptions 1, 2, and 4 ,

(i) the decision time for a $P$-bet leading to a preference reversal is longer than the decision time for a $P$-bet that does not lead to a preference reversal, i.e. $E\left[D T_{C} \mid C E_{\$}>C E_{P}, c(P, \$)=\right.$ $P]>E\left[D T_{C} \mid C E_{P}>C E_{\$}, c(P, \$)=P\right]$;

(ii) and the decision time for a \$-bet leading to a preference reversal is longer than the decision time for a $\$$-bet that does not lead to a preference reversal, i.e. $E\left[D T_{C} \mid C E_{P}>\right.$ $\left.C E_{\$}, c(P, \$)=\$\right]>E\left[D T_{C} \mid C E_{\$}>C E_{P}, c(P, \$)=\$\right]$.

The intuition for this result is again simple. Since the origin of reversals lies in the noise arising in the evaluation process, it is clear that reversals are more likely when utilities were close, and hence errors in the evaluation phase are more likely to reverse the order of the lotteries. Decisions where utilities are close are comparatively harder and hence take longer. In other words, reversals are more likely to involve hard choices than non-reversals, which leads to longer decision times. This proposition translates into the following experimental hypotheses.

H2a. The average decision time for predicted preference reversals is longer than the average decision time for comparable non-reversals (i.e. non-reversals where the $P$-bet is chosen).

H2b. The average decision time for unpredicted preference reversals is longer than the average decision time for comparable non-reversals (i.e. non-reversals where the $\$$-bet is chosen).

The next prediction is orthogonal to preference reversals. At the same time, it represents an a priori unexpected feature of the model and is hence especially valuable for its validation. It concerns decision times when the $\$$-bet was chosen given that it was priced higher compared to decision times when the $P$-bet was chosen given that it was priced higher.

Proposition 3. Under Assumptions 1, 2, 3, and 4, the decision time for a \$-bet that does not lead to a preference reversal is longer than the decision time for a P-bet that does not lead to a preference reversal, i.e. $E\left[D T_{C} \mid C E_{\$}>C E_{P}, c(P, \$)=\$\right]>E\left[D T_{C} \mid C E_{P}>C E_{\$}, c(P, \$)=P\right]$.

This result seems less intuitive. On the one hand, under overpricing, it is more likely that \$-bets will be priced higher than P-bets than the other way around. Hence, the probability that a hard (slow) \$-bet-choice will result in a non-reversal is larger than the probability that a hard $P$-bet-choice will result in a non-reversal. On the other hand, an easy (fast) $\$$-bet-choice is also more likely to result in a non-reversal than an easy $P$-bet-choice. The reason for this is that in the first case $u_{\$}>u_{p}$ and overpricing pushes the evaluations further apart, while in the second case $u_{\$}<u_{p}$ and overpricing pushes the evaluations together. Proposition 3 holds because the relative likelihood for a hard choice to lead to a non-reversal compared to the likelihood for an easy choice to lead to a non-reversal is larger for $\$$-bets than for P-bets. ${ }^{7}$ This result leads to our next experimental prediction.

H3. The average decision time for non-reversals where the $\$$-bet is chosen is longer than the average decision time for non-reversals where the $P$-bet is chosen.

\footnotetext{
7 In particular, the arguments in the proof of this result hold only for non-reversals. No analogous version of Proposition 3 for reversals can be established.
} 


\subsection{Order Effects and Preference Reappraisal}

Preference-reversal experiments include a pricing/evaluation phase and a choice phase. Thus far, the literature has remained silent on order effects, i.e. on whether there is any difference between experiments where the choice phase precedes the evaluation phase, and experiments where the order of the tasks is reversed. Preference reversals have been established using either of the two possible orderings.

We argue, however, that order effects need to be taken into account. The reason is that, as discussed above, imprecise preferences have been identified as one of the factors driving preference reversals. If preferences are imprecise, a large literature in psychology indicates that they might become more precise, or be generally altered, by the mere act of making choices. In the classical Free-Choice Paradigm (Brehm, 1956), subjects first face a rating (ranking) task, then a choice task, and finally another rating (ranking) task identical to the first one. The chosen options are usually evaluated more positively in the second rating (ranking) task while the options that were not chosen tend to be evaluated more negatively. ${ }^{8}$ According to Cognitive Dissonance Theory (Festinger, 1957), this happens because in the reevaluation phase subjects attempt to reduce the tension between the negative aspects of a chosen option and the positive aspects of an option that was not chosen. Self-Perception Theory (Bem, 1967), on the other hand, attributes this phenomenon to the fact that subjects learn their preferences better by making choices and hence ratings (rankings) in the second phase more closely resemble the "true preferences". This raises the question of whether preference reversals are affected by the order of valuation and choices. More precisely, if preference reappraisal occurs during the choice phase, there should be fewer preference reversals if the valuation task follows the choice task. This yields an additional hypothesis.

H4. Preference reversals are reduced if the valuation task follows the choice task, compared to the opposite ordering.

More generally, and in view of the discussion above, our expectation was that effects would in general be more clear when considering post-choice evaluations than when relying on prechoice ones. For example, if one relies on self-perception theory, classifying choices as reversals or non-reversals on the basis of pre-choice evaluations will result in some false classifications, effectively adding more noise to all measurements. However, since preference reversals have been established in the literature using both possible task orderings, we expected order effects to be subtle. The difference should be more clear for unpredicted reversals, because, if those are purely due to noise, any reduction of noise in the evaluation task should eliminate at least part of them.

\footnotetext{
8 Although this classical task has recently been shown to be affected by statistical biases (see Alós-Ferrer and Shi, 2015, and the references therein), improved versions of the task have meanwhile re-established the basic effect. See e.g. Alós-Ferrer et al. (2012).
} 


\section{Experiment 1: Preference Reversals and Decision Times}

The objective of our first experiment was to test our experimental predictions, as derived in Section 2, with regard to both choices and decision times. This would allow us to conclude that the combination of imprecise preferences in the evaluation phase and an overpricing phenomenon arising from the compatibility hypothesis is able to explain received evidence on preference reversals while at the same time fitting novel evidence on process data.

\subsection{Experimental Design and Procedures}

We followed a between-subject design comprised of three independent, consecutive single-decision making parts. The first and third phases were evaluation tasks, while the second, intermediate phase contained the choice task. This way, we can consider two kinds of preference reversals. On the one hand, we have "Price-Choice Reversals" which occur comparing the evaluations in the first phase and the choices in the second phase. On the other hand, we have "Choice-Price Reversals" which occur comparing the evaluations in the third phase and the choices in the second phase. Each of our Hypotheses H1 to H3 can be tested either on Price-Choice or Choice-Price reversals (or non-reversals), and we will report the results for both possibilities, keeping in mind that we expect clearer results for the Choice-Price ordering. Comparing both orderings allows us to test Hypothesis H4.

The stimuli were 40 different lotteries, which are presented in the online appendix. Each of the pairs in the choice task contained one $P$-bet and one $\$$-bet from this set of lotteries, with the former being defined by a high probability of winning a moderate amount of money and the latter being defined by a low probability of winning a high amount of money. ${ }^{9}$

We employed a pricing method for the evaluation of lotteries in phases 1 and 3. Participants were asked to state their minimum selling price for each of the 40 lotteries which were presented in fully randomized order ("State the lowest price for which you are just willing to sell the presented lottery."). Subjects were only allowed to state prices between $€ 2$ (the lower amount to win) and the higher amount to win. An example screen display for the pricing tasks is shown in the online appendix. The colors in the pie charts (green and blue) were counterbalanced across subjects. In phase two, the choice task, subjects faced the 20 lottery pairs sequentially and had to choose the lottery they would prefer to play out. See the online appendix for an example screen of the choice task. The order of the pairs and the onscreen position of the $P$-bet (i.e. left or right) was randomized. For each choice, we recorded the individuals' decision times as the time elapsed between the presentation of the lottery pair and clicking the button ("This lottery") underneath one of the two lotteries. After the three tasks, participants filled in a questionnaire containing various questions on their statistical knowledge, sociodemographic background, and personality attitudes.

\footnotetext{
9 Of the 20 lottery pairs, pairs 3 to 8 were such that the expected value of the $P$-bet was higher than the expected value of the $\$$-bet (with a difference between $€ 1.00$ to $€ 3.40$ ). Pairs 9 to 14 , which most closely resemble the ones commonly used in the literature, had roughly equal expected values. In pairs 15 to 20 , the $\$$-bet had a higher expected value than the $P$-bet (difference between $€ 1.60$ to $€ 4.80$ ). Finally, lottery pairs 1 and 2 were such that one bet dominated the other strictly and were only included as a basic rationality check. Only 2 out of 141 subjects chose one of the two strictly dominated lotteries in phase 2 . These two lottery pairs are therefore excluded from the analysis.
} 
All three tasks were incentivized. Payoffs were determined independently for each task after completion of the ex-post questionnaire to prevent spillover effects between tasks (e.g. through wealth effects). The two treatments in this experiment, $B D M$ and $\operatorname{OrdPM}$, differed only in the payment scheme used in the pricing tasks (phases 1 and 3). The former used a BDM payment scheme (Becker et al., 1964), and the latter a variant of the Ordinal Payment Method (Goldstein and Einhorn, 1987; Tversky et al., 1990; Cubitt et al., 2004). We included these two treatments to ensure that our results were robust with respect to the elicitation method.

The two schemes determined the payment in an evaluation task as follows. In the BDM treatment, after one of the 40 lotteries was picked at random the computer drew a price from a uniform distribution over the interval $] 2, A[$, where $A$ denotes the higher of the two amounts to win. If this price was higher than or equal to the price stated by the subject, the subject received this amount. If it was lower, the subject played the lottery and the payment was the realized outcome of that lottery. This was done separately for each pricing task. In the OrdPM treatment, two lotteries were chosen at random. The more highly priced lottery of the two was then played out and the realized outcome was the payoff for this phase (in case of a tie, the computer chose one at random). As in the BDM treatment, this procedure was conducted separately for the two pricing phases. Note that under the ordinal payment scheme, the absolute prices do not play a role, but only the induced ordering matters.

The payment method for the choice task in phase 2 was identical in both treatments. One of the 20 lottery pairs was picked at random, then the lottery the subject had chosen from this pair was played out and the realized outcome of that lottery was the payment for this round. The total payment a participant received in the experiment was the sum of realized payoffs in the three decision tasks.

\subsection{Procedures}

Before the experiment started, participants were briefly informed that the session consisted of three decision tasks, that payment for each task was partly determined by their decisions and partly by luck, that the tasks were paid independently of one another and that lotteries from each phase were not played out before the end of the experiment. In addition, four control questions had to be answered, using pencil and paper, before the start of the experiment to ensure that participants understood the concept of a lottery and its pie chart representation. Detailed instructions about each individual decision-making task (phase 1 to 3 ) and how payments would be determined in each phase were handed to the participants prior to the start of each phase.

The experiment was programmed in z-tree (Fischbacher, 2007). Participants were university students with majors other than psychology and economics. Each student participated in only one session. We conducted 7 sessions with a total of 141 participants (91 female). Of those, 67 were allocated to the BDM treatment and 74 to the OrdPM treatment. A session lasted about 2 hours with average earnings of $€ 24.76$ in the BDM treatment and of $€ 23.03$ in the OrdPM treatment. 


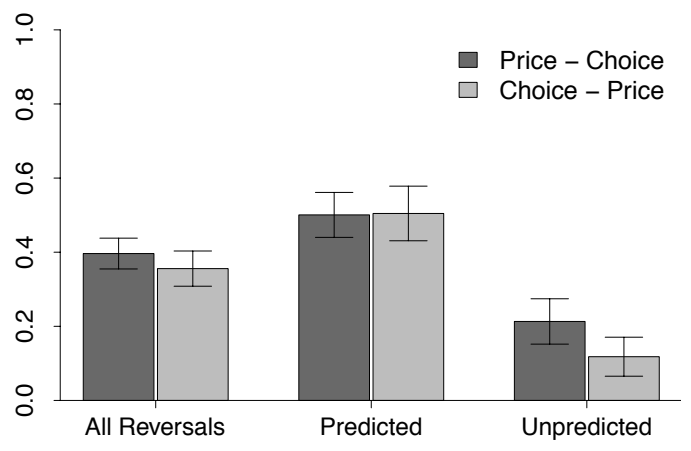

(a) BDM treatment

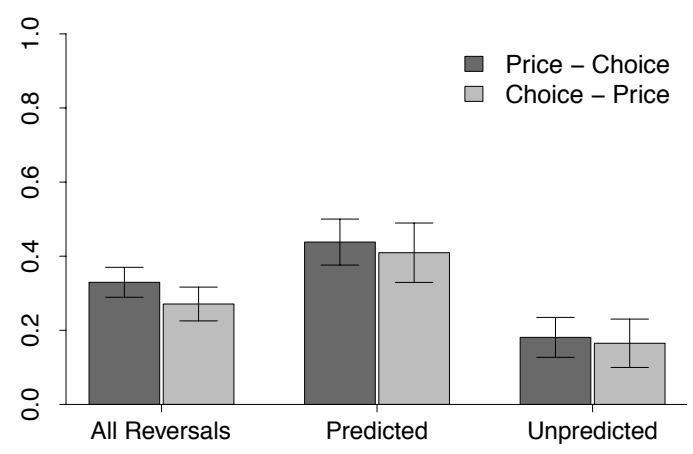

(b) OrdPM treatment

Figure 1: Average reversal rate per individual in Experiment 1.

Note: Reversal rates for the Price-Choice (dark bars) and Choice-Price (light bars) task orderings. Error bars depict the 95 percent confidence interval.

\subsection{Results of Experiment 1}

As a first illustration, Figure 1 displays the average reversal rates in the BDM and the OrdPM treatments in Experiment 1 (the percentage of $P$-bet choices in the BDM and OrdPM treatment was $69.6 \%$, and $65.6 \%$, respectively). The reversal rate is simply the number of reversals of any kind divided by the number of lottery pairs; the rate of predicted (unpredicted) reversals is computed as the number of predicted (unpredicted) reversals divided by the number of $P$-bet choices (\$-bet choices). It is already apparent from Figure 1 that the rate of predicted reversals is higher than that of unpredicted reversals, that measuring reversals with respect to postchoice attitudes decreases the rates, possibly by reducing noise, and that there might be some minor differences between treatments. We now proceed to test for these observations and our experimental hypotheses.

Predicted vs. unpredicted reversals (H1). We conducted two-sided Wilcoxon Signed-Rank (hereafter WSR) tests to assess whether the rate of predicted reversals was higher than the rate of unpredicted reversals. We computed the predicted and unpredicted preference reversal rates for each subject individually. The rates for predicted reversals were significantly higher than the rates of unpredicted reversals for both treatments and both possible task orderings (BDM PriceChoice, $N=60, z=4.170, p<0.0001$; BDM Choice-Price, $N=60, z=5.140, p<0.0001$; OrdPM Price-Choice, $N=69, z=4.585, p<0.0001$; OrdPM Choice-Price, $N=69, z=3.595$, $p<0.0005) .{ }^{10}$ This confirms Hypothesis H1.

Order effects (H4). In the BDM treatment, the rate of unpredicted reversals was significantly lower when prices were elicited after choices (Choice-Price) than when they were elicited before choices (Price-Choice) (two-sided WSR test, $N=61, z=-3.014, p=0.003$ ). In the OrdPM treatment, the same effect was observed $(N=67, z=-2.70, p=0.007)$ after exclusion of four

\footnotetext{
10 The tests for reversal rates include of course only the participants for which both rates can be computed. For instance, if a participant never chose a \$-bet, no rate of unpredicted reversals can be computed.
} 


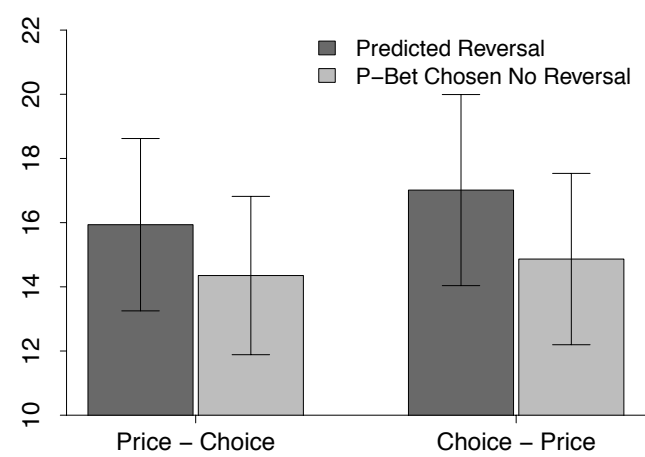

(a) BDM treatment, Exp. 1

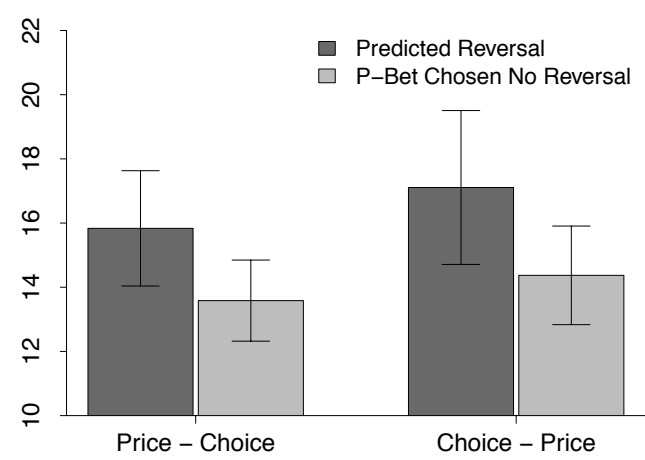

(c) OrdPM treatment, Exp. 1

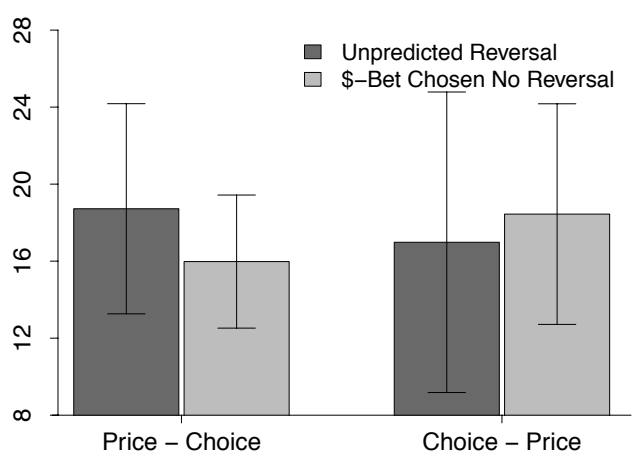

(b) BDM treatment, Exp. 1

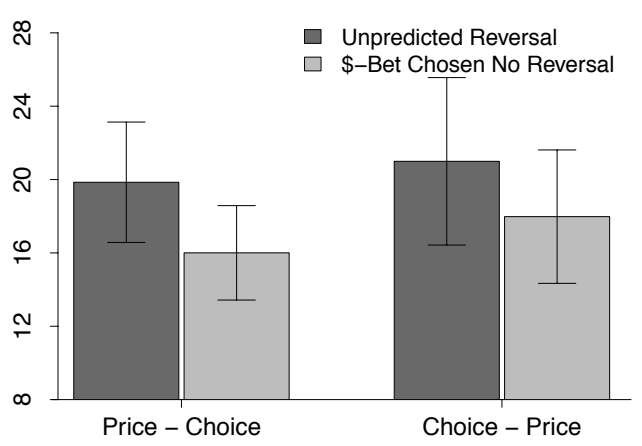

(d) OrdPM treatment, Exp. 1

Figure 2: Average decision time per individual in choice task of Experiment 1.

Note: Predicted reversals are compared to non-reversals where the $P$-bet was chosen, unpredicted reversals to non-reversals where the $\$$-bet was chosen. Error bars depict the 95 percent confidence interval.

extreme data points. ${ }^{11}$ There were no significant differences in the rate of predicted reversals (BDM, $N=66, z=-0.465, p=0.642$; OrdPM, $N=72, z=1.090, p=0.276)$. Since unpredicted reversals are essentially due to noise, this is consistent with the interpretation that measuring reversals through post-choice evaluations reduces noise.

Treatment effects (reversals). We compared the individual reversal rates across treatments using Mann-Whitney-U (MWU) tests. We found a significantly lower rate of predicted reversals when prices are elicited after choices but not when prices are elicited before choices (Price-Choice, $z=1.49, p=0.135$; Choice-Price, $z=0.42, p=0.058)$. There were, however, no significant differences for unpredicted reversals (Price-Choice, $z=1.90, p=0.678$; Choice-Price, $z=-0.88$, $p=0.380)$.

\footnotetext{
11 Four participants made only one \$-bet choice yielding an unpredicted reversal in choice-price but not in price-choice, resulting in increases of $100 \%$ in the reversal rate. Including these four data points, the effect is not significant anymore $(N=71, z=-1.339, p=0.181)$.
} 


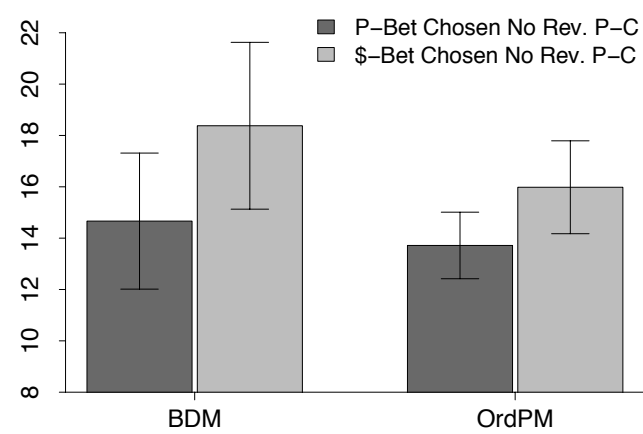

(a) Price-Choice

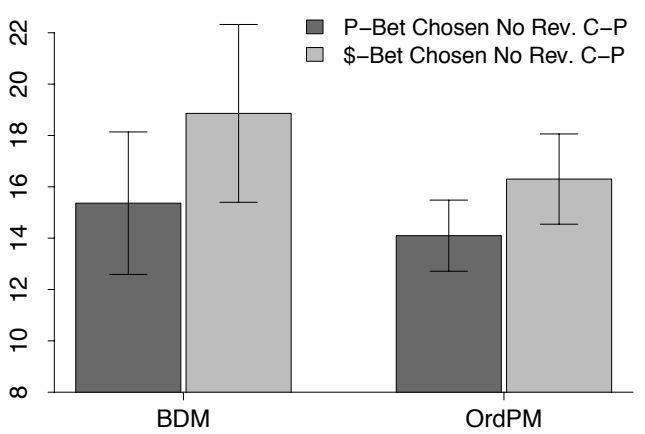

(b) Choice-Price

Figure 3: Average non-reversal decision time per individual in choice task of Experiment 1.

Note: Choices classified as non-reversals according to the indicated task ordering, Price-Choice (left) and Choice-Price (right). Error bars depict the 95 percent confidence interval.

Decision times and reversals (H2). Figure 2 displays the decision times for reversals and comparable non-reversals for both treatments and both task orderings. Each type of reversal is compared with the correct counterfactual, i.e. predicted reversals are compared with nonreversals where the $P$-bet was chosen, and unpredicted reversals with non-reversals where the $\$$-bet was chosen.

Two-sided WSR tests confirmed that predicted reversals involved significantly longer decision times than comparable non-reversals, both for Price-Choice and for Choice-Price, both for the BDM (Price-Choice $N=61, z=2.758, p=0.006$; Choice-Price, $N=54, z=3.625, p<0.0005$ ) and the OrdPM treatments (Price-Choice, $N=66, z=2.894, p=0.004$; Choice-Price, $N=57$, $z=2.987, p=0.003) .{ }^{12}$ Unpredicted reversals were also associated with significantly longer decision times in the OrdPM treatment (Price-Choice, $N=39, z=2.854, p=0.004$; ChoicePrice, $N=22, z=1.883, p=0.060$ ), but there were no significant differences for unpredicted reversals in the BDM treatment (Price-Choice, $N=31, z=0.950, p=0.342$; Choice-Price, $N=17, z=-0.947, p=0.344)$.

Decision times and non-reversals (H3). Non-reversals were clearly slower when the $\$$-bet was chosen than when the $P$-bet was chosen (see Figure 3). The difference was highly significant independently of whether choices were declared non-reversals according to pre-choice or postchoice evaluations, for both the BDM treatment (Price-Choice, $N=56, z=3.242, p=0.001$; Choice-Price, $N=51, z=2.995, p=0.003$ ) and the OrdPM treatment (Price-Choice, $N=64$, $z=-3.681, p<0.0005$; Choice-Price, $N=59, z=-3.204, p=0.001)$.

\footnotetext{
12 Every test on decision times was conducted for the population of subjects for which the involved average decision times could be computed. For instance, if a subject did not display any unpredicted reversal, no decision time can be computed for this category.
} 


\subsection{Regression Analysis for Experiment 1}

We conducted a random-effects panel regression analysis (with standard errors clustered at the subject level) to further investigate the relation between preference reversals and decision times, and to further test our hypotheses while controlling for a number of natural variables, e.g. individual and lottery-pair covariates. Since decision times are always positive, we used the log of decision times $(\log D T)$ as the dependent variable. For each treatment, we report a regression including a dummy variable for Price-Choice reversals and an analogous one with a dummy variable for Choice-Price reversals (see Table 1). We also ran a number of additional regressions and found the main effects to be robust (in magnitude and significance) to the inclusion or exclusion of additional control variables.

The regressions include dummies for choices which were part of reversals, for $\$$-bet choices, and the interaction thereof. Hence we can make any comparison among reversals and nonreversals where the $\$$-bet or the $P$-bet was chosen, either directly through specific regression coefficients or via appropriate postestimation tests, which are also reported in the table.

Predicted reversals vs. non-reversals. Hypothesis H2a states that decision times for predicted preference reversals should be longer on average than decision times for comparable nonreversals, i.e. non-reversals where the $P$-bet was chosen. Since a $\$$-choice dummy is included, the comparison between predicted reversals and non-reversals where the $P$-bet was chosen corresponds to the reversal dummy in the regression, which is highly significant and positive for both regressions for the OrdPM treatment, and for the Choice-Price regression for the BDM treatment. This indicates that predicted reversals took longer than comparable non-reversals, confirming Hypothesis H2a.

Unpredicted reversals vs. non-reversals. Hypothesis $\mathrm{H} 2 \mathrm{~b}$ states that unpredicted reversals should take longer than non-reversals where the $\$$-bet was chosen. The difference between both types of choices corresponds to $\beta_{\text {Reversal }}+\beta_{\$-\text { Choice } \times \text { Reversal }}$, which is highly significant and positive in both regressions for the OrdPM, confirming Hypothesis 2b. However, the postestimation tests are not significant for the BDM treatment.

Comparison of non-reversals. According to Hypothesis H3, non-reversals where the \$-bet was chosen should take longer than non-reversals where the $P$-bet was chosen. Since reversals dummies are included, this comparison corresponds to the $\$$-choice dummy, which is highly significant and positive for all four regressions. Hence, conditional on the absence of a preference reversal, \$-bet choices took longer, confirming Hypothesis H3.

Controls: Lotteries. We included a number of covariates in order to control for differences in the lottery pairs. The absolute value of the difference in expected values of the $P$-bet and the $\$$-bet (DiffEV) had a weakly significant negative effect in both treatments. We further included the absolute difference in the prices stated for the lotteries in phases one and three (StatedDiff1, StatedDiff-3) as a rough measure of how similar (or different) the participant viewed the lotteries within a pair. Both were highly significant in the OrdPM treatment, but essentially not significant in the BDM treatment.

The ratio of the two higher amounts to win in the $\$$-bet and the $P$-bet (Ratio) had a significant positive effect in both treatments. This effect has a direct interpretation. Given our 
Table 1: Random-effects panel regressions for decision times, Experiment 1.

\begin{tabular}{|c|c|c|c|c|}
\hline $\begin{array}{l}\text { Treatment } \\
\text { Order }\end{array}$ & $\begin{array}{l}\text { BDM } \\
\text { P-C }\end{array}$ & $\begin{array}{l}\text { BDM } \\
\text { C-P }\end{array}$ & $\begin{array}{l}\text { OrdPM } \\
\text { P-C }\end{array}$ & $\begin{array}{l}\text { OrdPM } \\
\text { C-P }\end{array}$ \\
\hline ReversalPC & $\begin{array}{r}0.018 \\
(0.029)\end{array}$ & & $\begin{array}{l}0.078^{* * *} \\
(0.025)\end{array}$ & \\
\hline ReversalCP & & $\begin{array}{l}0.083^{* * *} \\
(0.031)\end{array}$ & & $\begin{array}{l}0.109^{* * *} \\
(0.027)\end{array}$ \\
\hline \$-Choice & $\begin{array}{l}0.127^{* * *} \\
(0.054)\end{array}$ & $\begin{array}{l}0.155^{* * *} \\
(0.031)\end{array}$ & $\begin{array}{l}0.126^{* * *} \\
(0.027)\end{array}$ & $\begin{array}{l}0.151^{* * *} \\
(0.026)\end{array}$ \\
\hline $\begin{array}{l}\$ \text {-Choice } \\
\quad \times \text { ReversalPC }\end{array}$ & $\begin{array}{r}-0.097 \\
(0.073)\end{array}$ & & $\begin{array}{l}0.107^{* *} \\
(0.052)\end{array}$ & \\
\hline $\begin{array}{l}\text { \$-Choice } \\
\quad \times \text { ReversalCP }\end{array}$ & & $\begin{array}{l}-0.189^{* *} \\
(0.095)\end{array}$ & & $\begin{array}{r}0.058 \\
(0.064)\end{array}$ \\
\hline DiffEV & $\begin{array}{l}-0.023^{*} \\
(0.012)\end{array}$ & $\begin{array}{l}-0.022^{*} \\
(0.012)\end{array}$ & $\begin{array}{l}-0.021^{* *} \\
(0.009)\end{array}$ & $\begin{array}{l}-0.023^{* *} \\
(0.009)\end{array}$ \\
\hline Ratio & $\begin{array}{l}0.041^{* * *} \\
(0.011)\end{array}$ & $\begin{array}{l}0.036^{* * *} \\
(0.011)\end{array}$ & $\begin{array}{l}0.038^{* * *} \\
(0.008)\end{array}$ & $\begin{array}{l}0.038^{* * *} \\
(0.008)\end{array}$ \\
\hline StatedDiff-1 & $\begin{array}{r}-0.010 \\
(0.007)\end{array}$ & $\begin{array}{r}-0.009 \\
(0.007)\end{array}$ & $\begin{array}{l}-0.008^{* *} \\
(0.003)\end{array}$ & $\begin{array}{l}-0.009^{* * *} \\
(0.003)\end{array}$ \\
\hline StatedDiff-3 & $\begin{array}{r}-0.010 \\
(0.006)\end{array}$ & $\begin{array}{l}-0.011^{*} \\
(0.006)\end{array}$ & $\begin{array}{l}-0.011^{* * *} \\
(0.003)\end{array}$ & $\begin{array}{l}-0.011^{* * *} \\
(0.003)\end{array}$ \\
\hline Round & $\begin{array}{l}-0.008^{* * *} \\
(0.003)\end{array}$ & $\begin{array}{l}-0.008^{* * *} \\
(0.003)\end{array}$ & $\begin{array}{l}-0.005^{* * *} \\
(0.002)\end{array}$ & $\begin{array}{l}-0.005^{* * *} \\
(0.002)\end{array}$ \\
\hline Female & $\begin{array}{l}-0.286^{* *} \\
(0.113)\end{array}$ & $\begin{array}{l}-0.290^{* *} \\
(0.113)\end{array}$ & $\begin{array}{l}-0.156^{* *} \\
(0.072)\end{array}$ & $\begin{array}{l}-0.151^{* *} \\
(0.071)\end{array}$ \\
\hline Position & $\begin{array}{r}0.016 \\
(0.023)\end{array}$ & $\begin{array}{r}0.015 \\
(0.023)\end{array}$ & $\begin{array}{l}-0.012 \\
(0.019)\end{array}$ & $\begin{array}{r}-0.009 \\
(0.019)\end{array}$ \\
\hline Color & $\begin{array}{r}-0.021 \\
(0.111)\end{array}$ & $\begin{array}{r}-0.019 \\
(0.112)\end{array}$ & $\begin{array}{r}0.088 \\
(0.070)\end{array}$ & $\begin{array}{r}0.084 \\
(0.068)\end{array}$ \\
\hline Constant & $\begin{array}{c}2.666^{* * *} \\
(0.106)\end{array}$ & $\begin{array}{c}2.649^{* * *} \\
(0.105)\end{array}$ & $\begin{array}{c}2.563^{* * *} \\
(0.073)\end{array}$ & $\begin{array}{c}2.556^{\text {*** }} \\
(0.072)\end{array}$ \\
\hline Nr. Obs. & 1340 & 1340 & 1480 & 1480 \\
\hline Nr. Groups & 67 & 67 & 74 & 74 \\
\hline R2-Overall & 0.103 & 0.101 & 0.118 & 0.119 \\
\hline Wald test & 0.000 & 0.000 & 0.000 & 0.000 \\
\hline $\begin{array}{l}\text { Postestimation tests } \\
\text { Reversal } \\
\quad+(\$ \text {-Choice } \times \text { Reversal })\end{array}$ & $\begin{array}{r}-0.079 \\
(0.072)\end{array}$ & $\begin{array}{r}-0.106 \\
(0.094)\end{array}$ & $\begin{array}{l}0.185^{* * *} \\
(0.045)\end{array}$ & $\begin{array}{l}0.167^{* * *} \\
(0.056)\end{array}$ \\
\hline
\end{tabular}

Note: All regressions are random-effects panel estimations, with log decision time as dependent variable. Standard errors in parentheses. ${ }^{* * *} p<0.01,{ }^{* *} p<0.05,{ }^{*} p<0.1$. 
construction of lottery pairs, a larger ratio translates into the high amount to win being much larger in the $\$$-bet than in the $P$-bet, which makes the $\$$-bet more attractive on the monetary domain. At the same, the probability to win the high amount is much larger for the $P$-bet than for the $\$$-bet, which makes the $P$-bet more attractive on the probability domain. This tradeoff leads to "more difficult" decisions which in turn lead to longer response times.

Other controls. Decision time measurements in repeated tasks usually capture a learning effect as participants gain familiarity with the interface. We controlled for this effect by including the round in which the choice was made as a regressor (Round). This was significantly negative in both treatments. A dummy variable controlling for gender (Female) was also significant in both treatments. Finally, we controlled for onscreen position (Position) of the P-bet and the $\$$-bet and for the colors used in the pie-chart (Color) to verify that these factors did not influence the results. As expected, these variables never had significant effects.

\subsection{Discussion of Experiment 1}

The analysis of the data confirms our predictions as derived in Section 2. First, the rate of predicted reversals is clearly higher than the rate of unpredicted ones, in agreement with previous experiments. Second, preference reversals appear to involve longer decision times. This effect is clear for an ordinal-based elicitation of prices; in the BDM treatment, the effect is also present albeit less pronounced. Third, in both treatments we found that $\$$-bet choices which are part of non-reversals take significantly longer than $P$-bet choices part of non-reversals.

In view of the evidence, we conclude that the data is compatible with the idea that preference reversals arise from the combination of two factors. First, as pointed out by Schmidt and Hey (2004) and Butler and Loomes (2007), monetary valuations of lotteries are typically imprecise, and hence preference elicitation through pricing tasks is much noisier than actual choices. Second, as summarized by the compatibility hypothesis (Tversky et al., 1988, 1990), the use of pricing tasks causes an overpricing phenomenon which anchors up the evaluation of bets with a saliently high monetary outcome. These observations produce testable hypotheses for both behavioral data and decision times once we incorporate the observation that easier choices (where the alternatives are farther away from indifference) are faster (e.g. Wilcox, 1993; Shultz et al., 1999; Moffatt, 2005).

Regarding ordering effects, we observe small but systematic differences suggesting that a Price-Choice ordering, where the evaluation task precedes actual choices, might be noisier than the opposite order, hence producing both higher reversal rates and slightly less clear effects. This is compatible with self-perception theory (Bem, 1967), which holds that actual choices serve as "self-signals" that help reduce noise in future evaluations of alternatives.

Last, we observe small but definite treatment effects, pointing out that price evaluations conducted through the BDM "price-list" scheme might be noisier than those conducted according to a more intuitive, ordinal-like scheme. This is reflected by the fact that reversal rates (especially of unpredicted reversals, which are presumably due to noise) are higher in the BDM case. It is also compatible with the general observation that effects are often more clearly observed in the OrdPM treatment than in the BDM one. 


\section{Experiment 2: Eliminating Reversals}

The objective of our second experiment was twofold. First, we wanted to show that the overpricing phenomenon can be next to eliminated by using ordinal, ranking-based evaluation tasks. Second, this manipulation would allow us to disentangle the two building blocks of our model. The absence of the overpricing phenomenon should result in a reduction of predicted preference reversals, while the assumption of imprecise preferences still delivers predictions on decision times.

\subsection{Motivation and Hypotheses}

In our first experiment we found that the method used to elicit participants' minimum selling prices affects the rate of preference reversals. According to the compatibility hypothesis, predicted reversals are more frequent because participants focus more on monetary outcomes when their preferences are elicited through prices. Notably, predicted preference reversals were also more frequent in the OrdPM treatment, where the use of prices in the evaluation task was simple framing, with no direct monetary consequences. This raises the natural hypothesis that the overpricing phenomenon predicted by the compatibility hypothesis arises due to a price-based, cardinal framing (i.e., a "rating task") in the evaluation phases. As a consequence, moving away from a cardinal evaluation task towards a more natural, ordinal-based one (a "ranking task") should greatly reduce the rate of predicted reversals (given that behavior in the choice phase does not change).

Specifically, suppose that, by employing a ranking-based evaluation task, we were able to shut down the decision process responsible for the overpricing phenomenon. In terms of the model in Section 2, this would imply $K=0$ in Assumption 3. It is easy to revisit our theoretical predictions and derive new experimental hypotheses for such a situation. First, Proposition 1 crucially depends on Assumption 3, and hence we would not expect Hypothesis H1 to hold in this setting. Although from the point of view of the model we would expect no differences in reversal rates, this rests upon the implicit assumption that there is no other (second-order) latent process causing unpredicted reversals. Even if this was the case, a conservative hypothesis derived from our theoretical analysis is that the rate of predicted preference reversals should be greatly reduced in comparison to treatments with price-framed evaluations.

H5. The rate of predicted preference reversals is lower if ordinal, ranking-based evaluation tasks are used than if rating-based tasks are used.

Hypothesis $\mathrm{H} 5$ is also in line with previous findings in the literature. Ordinal-based evaluation tasks reduce the difference between the choice task and the evaluation task. For example Bateman et al. (2007) show that a ranking task reduces predicted reversals, although the ranking task they use is not directly comparable to ours (see Section 4.6).

The first decision-times predictions spelled out in Proposition 2 do not depend on Assumption 3. Hence, independently of whether evaluation tasks are based on ratings or rankings, we would expect Hypotheses H2a/b to hold. 
H6a/b. Even if ordinal, ranking-based evaluation tasks are used, choices associated with predicted preference reversals take longer than $P$-bet choices associated with non-reversals, and choices associated with unpredicted preference reversals take longer than $\$$-bet choices associated with non-reversals.

Proposition 3 depends on Assumption 3. If $K=0$, we would a priori expect no differences in the decision times associated with non-reversals where the $P$-bet or the $\$$-bet was chosen.

H7. If ordinal, ranking-based evaluation tasks are used, the average decision time for nonreversals where the $P$-bet is chosen is not different from the average decision time for nonreversals where the $\$$-bet is chosen.

\subsection{Design of Experiment 2}

The setup of the second experiment was identical to Experiment 1, with the exception of the evaluation tasks. We used two different ranking-based tasks, meant to shut down the overpricing decision processes, and one BDM task as a control. In each of the three treatments, presentation of lotteries was such that participants faced a total of six blocks consisting of six lotteries each, i.e. a total of 18 pairs. ${ }^{13}$ In the Rank-Unframed treatment, we used a purely ranking-based task. Participants were asked to assign ranks (from most preferred to least preferred) to the lotteries according to how much they would like to play each lottery, separately for each block. Most importantly, we did not make any reference to prices (see the online appendix for an example screen display of the two ranking treatments). In this sense, the task was unframed. The RankFramed treatment was programmed identically. The only difference was in the experimental instructions. Participants were asked to rank the lotteries (from 1 to 6) according to their minimum selling price, separately for each block. However, they were not asked to type in or otherwise state the prices, but merely to think about them and use them for the ranking. Finally, in the BDM2 treatment, participants had to complete a pricing task that was identical to the one in the BDM treatment in Experiment 1, with the only exception that (for comparability with the other treatments) lotteries were presented one after another in three blocks of six lotteries each. Again, colors and onscreen positions of the lotteries were completely randomized in all treatments.

As in Experiment 1, all three tasks were incentivized and payoffs for each task were determined independently. Payoffs for the evaluation task of the BDM2 and the choice tasks of all three treatments were determined in the same way as in Experiment 1. Payoffs for the evaluation phases for Treatments Rank-Unframed and Rank-Framed were determined as follows. First, the computer picked one of the six blocks at random. From the six lotteries contained in that block, the computer again randomly picked two. The one that had been ranked higher by the participant was then played out and the participant received the outcome of that lottery as payment for that round. In all three treatments, payments were determined and presented to participants only after all three tasks had been completed.

\footnotetext{
13 We used 18 of the lottery pairs from Experiment 1, excluding the two pairs which contained stochastically dominated lotteries.
} 
Since in both ranking treatments there was no actual "pricing" task, we will refer to the two possible task orderings for these treatments as "Rank-Choice" and "Choice-Rank". ${ }^{14}$

\subsection{Procedures}

We followed the same procedures as in Experiment 1. We conducted 12 sessions with a total of 215 participants (102 female). Of those, 73 were allocated to the Rank-Unframed treatment, 73 to the Rank-Framed treatment, and 69 to the BDM2 treatment. Sessions in the Rank-Unframed treatment lasted roughly an hour with average earnings of $€ 23.36$. Sessions in the Rank-Framed treatment lasted one hour and 20 minutes with average earnings of $€ 24.07$, while sessions in the BDM2 treatment lasted about 2 hours with average earnings of $€ 28.44$.

\subsection{Results of Experiment 2}

Figure 4 shows the average reversal rates for all three treatments for both Price/Rank-Choice and Choice-Price/Rank reversals (the percentage of $P$-bet choices in the BDM2, Rank-Framed, and Rank-Unframed treatment were $69.4 \%, 66.7 \%$, and $66.2 \%$, respectively). As before, the rate of predicted (unpredicted) reversals is computed as the number of predicted (unpredicted) reversals divided by the number of $P$-bet choices ( $\$$-bet choices). The basic trends are already apparent. The rate of predicted reversals was enormously reduced in both ranking treatments to the extent of dropping below the rate of unpredicted reversals. Measuring reversals with respect to post-choice attitudes does not have an effect on the reversal rates.

$B D M$ replication. The first observation is that, as expected, there is no qualitative difference between the results of Treatment BDM2 and Treatment BDM of Experiment 1. For instance, in Treatment BDM2 the rates of predicted reversals are significantly higher than the rates of unpredicted reversals (Price-Choice, $N=68, z=4.495, p<0.0001$; Choice-Price, $z=4.585$, $p<0.0001)$.

Reduction of predicted reversals (H5). Kruskal-Wallis tests confirmed that the rate of predicted reversals was significantly different across treatments for both task orderings (Price/RankChoice, $\chi^{2}=59.239, \mathrm{df}=2, p<0.0001$; Choice-Price/Rank, $\left.\chi^{2}=60.5983, \mathrm{df}=2, p<0.0001\right)$. To confirm that the differences were between the ranking treatments and the control BDM2 treatment, we conducted two-sided MWU tests with Holm-Bonferroni correction to account for multiple comparisons ( $p$-values below are the adjusted values). Both ranking treatments generated significantly lower predicted reversal rates than the BDM2 treatment (Rank-Framed Price/RankChoice, $z=6.01, p<0.0001$; Rank-Framed Choice-Price/Rank, $z=5.75, p<0.0001$; Rank-Unframed Price/Rank-Choice, $z=7.19, p<0.0001$; Rank-Unframed Choice-Price/Rank, $z=7.25, p<0.0001)$. The difference in the rate of predicted reversals across both ranking treatments was not significant for the Rank-Choice ordering $(z=-0.81, p=0.414)$, but for the Choice-Rank ordering the rate of predicted reversals was significantly lower in the Rank-

\footnotetext{
14 Our experimental setup does not allow distinguishing between the scale compatibility hypothesis and reference dependence (Sugden, 2003; Schmidt et al., 2008; Lindsay, 2013) as an explanation for the overpricing of $\$$-bets. This is due to the fact that when asking subjects to think about their minimum selling price (be it in a pricing or a framed ranking task) both effects would be present whereas in an unframed ranking task both effects would be absent.
} 


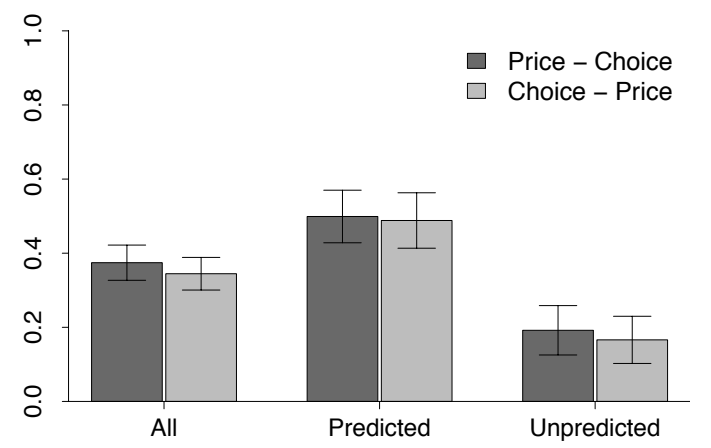

(a) BDM treatment

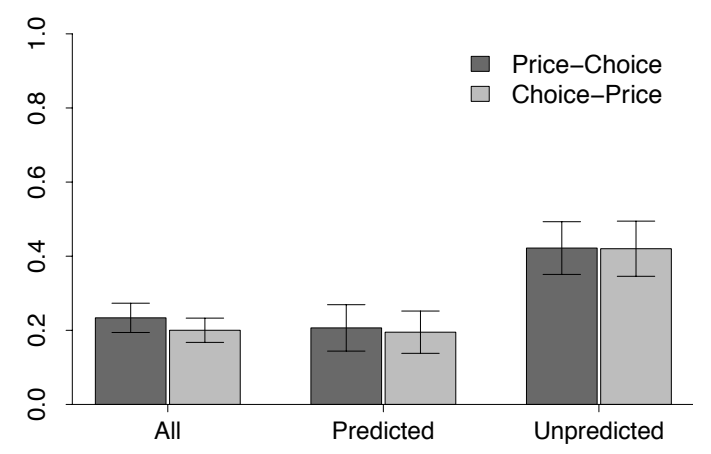

(b) Rank-Framed treatment

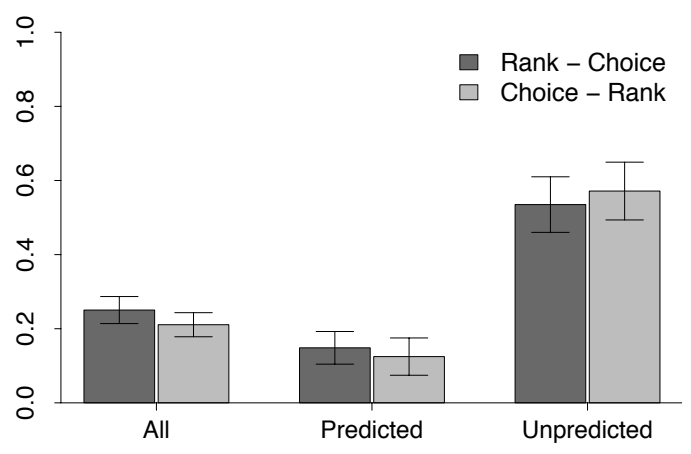

(c) Rank-Unframed treatment

Figure 4: Average reversal rate per individual in Experiment 2.

Note: Reversal rates for the Price/Rank-Choice (dark) and Choice-Price/Rank (light) orderings. Error bars depict the 95 percent confidence interval.

Unframed treatment than in the Rank-Framed treatment $(z=-2.29, p=0.022)$. This last result agrees with the idea that the Rank-Unframed treatment goes one step further in the elimination of the overpricing process than a ranking-based but still price-framed approach.

Reversal of the standard reversal pattern. In the ranking treatments, the usual pattern of reversals was reversed, with unpredicted reversals becoming more prominent than predicted ones (Figure 4). Two-sided WSR tests confirm this observation. The rate of unpredicted reversals is significantly higher than the rate of predicted reversal rates in both ranking treatments independent of the task ordering (Rank-Framed Rank-Choice, $N=66, z=3.47, p=0.001$; RankFramed Choice-Rank, $N=66, z=3.17, p=0.002$; Rank-Unframed Rank-Choice, $N=68$, $z=5.27, p<0.0001$; Rank-Unframed Choice-Rank, $N=66, z=5.71, p<0.001$ ).

Order effects (H4). In contrast to Experiment 1, we found no significant differences in neither the rate of unpredicted reversals (BDM2, $N=68, z=0.918, p=0.359$; Rank-Framed, $N=66$, $z=0.235, p=0.814$; Rank-Unframed, $N=70, z=-0.474, p=0.636)$ nor in the rate of predicted reversals (BDM2, $N=69, z=503, p=0.615$; Rank-Framed, $N=73, z=0.453$, 
$p=0.651$; Rank-Unframed. $N=71, z=1.479, p=0.139)$ when prices were elicited after choices compared to when they were elicited before choices.

Decision times and reversals (H2/H6). Figure 5 displays the decision times for reversals and comparable non-reversals for all three treatments and both task orderings, comparing each type of reversal with the appropriate non-reversals. Two-sided WSR tests confirmed that predicted reversals involved longer decision times than comparable non-reversals, both for Price/RankChoice and for Choice-Price/Rank, for all treatments. For both ranking treatments, the differences were highly significant (Rank-Framed Rank-Choice, $N=42, z=3.551, p<0.0005$; Rank-Framed Choice-Rank, $N=45, z=2.743, p=0.006$; Rank-Unframed Rank-Choice, $N=43, z=2.614, p=0.009$ ); Rank-Unframed Choice-Rank, $N=34, z=3.163, p=0.002)$. This confirms that the decision times effect predicted by our model, which is independent of the overpricing assumption, is still present under ordinal (ranking) evaluation tasks. In the case of the BDM2 treatment, the test missed significance for the Price-Choice ordering $(N=64$, $z=1.595, p=0.111)$, but the difference was significant for Choice-Price $(N=58, z=3.004$, $p=0.003)$.

For both ranking treatments unpredicted reversals were again significantly slower than comparable non-reversals independently of task ordering (Rank-Framed Rank-Choice, $N=49$, $z=2.875, p=0.004$; Rank-Framed Choice-Rank, $N=45, z=3.014, p=0.003$; RankUnframed Rank-Choice, $N=49, z=1.930, p=0.054)$; Rank-Unframed Choice-Rank, $N=47$, $z=3.656, p<0.0005)$. In the BDM2 treatment the decision time differences were not significant for the Price-Choice ordering $(N=30, z=1.131, p=0.258)$, but unpredicted reversals were significantly slower for the Choice-Price ordering $(N=23, z=1.992, p=0.046)$.

Decision times and non-reversals $\left(\mathrm{H}_{3} / \mathrm{H}^{7}\right)$. Treatment BDM2 successfully replicated the finding that non-reversals are slower when the $\$$-bet is chosen than when the $P$-bet is chosen, as predicted in Hypothesis H3 (WSR tests; Price-Choice, $N=60, z=1.984, p=0.047$; Choice-Price, $N=58, z=2.609, p=0.009)$. However, for ranking treatments we expected no differences (Hypothesis H7). There is still a significant difference for the Rank-Choice ordering (Rank-Framed, $N=57, z=1.835, p=0.066$; Rank-Unframed, $N=54, z=1.825, p=0.068$ ), but there is clearly no significant difference for the classification according to the Choice-Rank ordering (Rank-Framed, $N=55, z=0.733, p=0.463$; Rank-Unframed, $N=48, z=0.385$, $p=0.701)$. Figure 6 illustrates these results.

Decision times in the Rank-Unframed Treatment. All decisions in the Rank-Unframed treatment were significantly quicker than in the other two treatments (Figures 5 and 6). The difference is substantial: the median decision time over all choices was $13.41 \mathrm{~s}$ in BDM2, $12.52 \mathrm{~s}$ in Rank-Framed, and only $9.61 \mathrm{~s}$ in Rank-Unframed. This is remarkable, because the treatments differed only in the evaluation phases, and the choice phases in which the decision times were measured were identical. We will discuss this observation in detail below.

A Kruskal-Wallis test confirmed that decision times were significantly different across treatments $\left(\chi^{2}=35.545, \mathrm{df}=2, p<0.0001\right)$. Two-sided MWU tests with Holm-Bonferroni correction to account for multiple comparisons showed that decisions were faster in the RankUnframed treatment than in the other treatments (BDM2, $z=-5.722, p<0.0001$; Rank- 


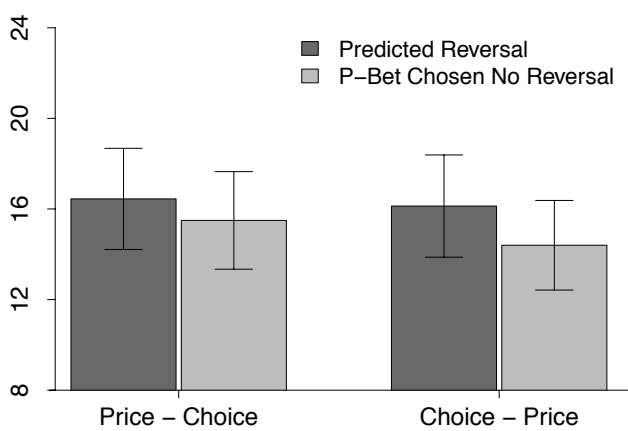

(a) BDM treatment

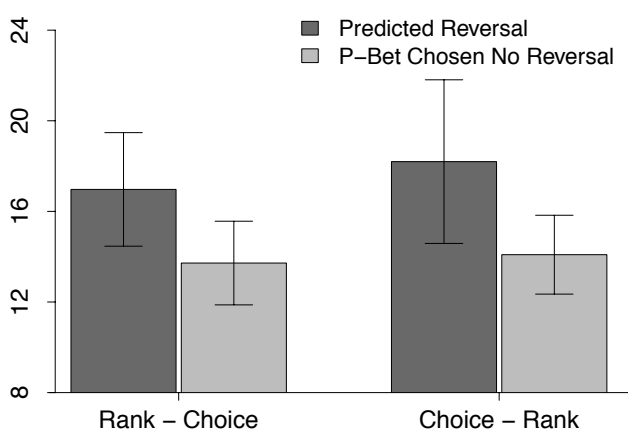

(c) Rank-Framed treatment

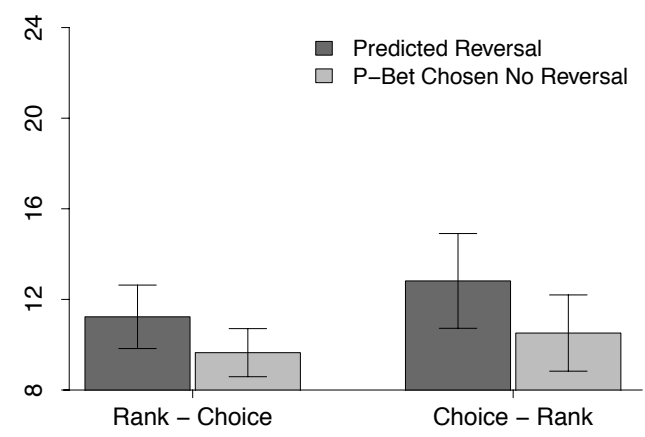

(e) Rank-Unframed treatment

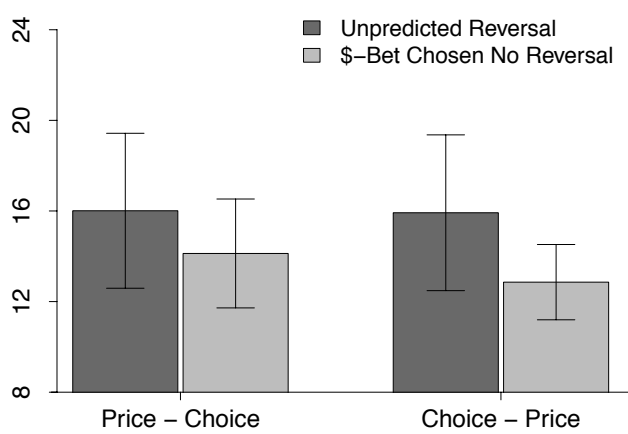

(b) BDM treatment

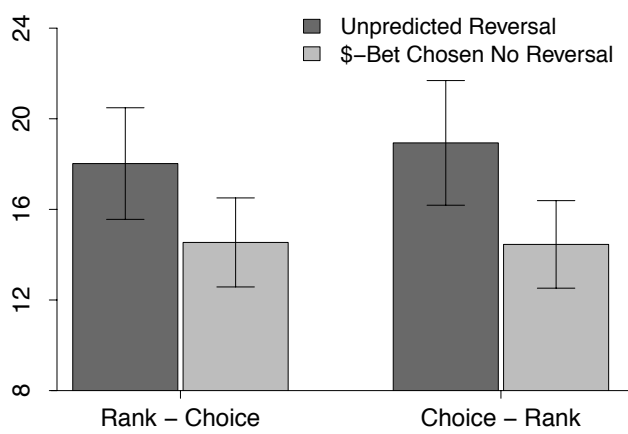

(d) Rank-Framed treatment

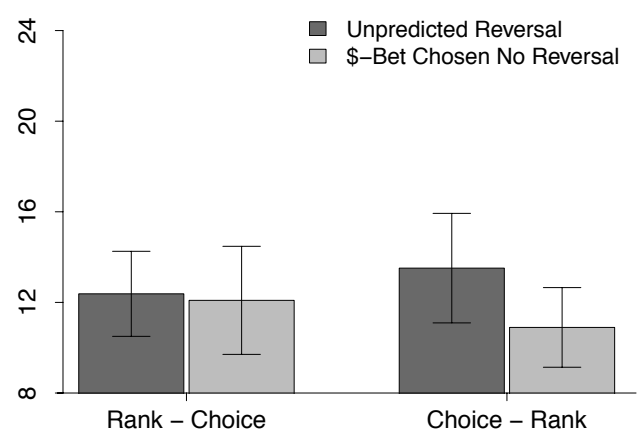

(f) Rank-Unframed treatment

Figure 5: Average decision time per individual in the choice task in Experiment 2.

Note: Predicted reversals are compared to non-reversals where the $P$-bet was chosen, unpredicted reversals to non-reversals where the $\$$-bet was chosen. Error bars depict the 95 percent confidence interval.

Framed, $z=-4.225, p<0.0001){ }^{15}$

\footnotetext{
15 The difference between decision times in treatments Rank-Framed and BDM2 missed significance, $z=$ $-1.596, p=0.111$.
} 


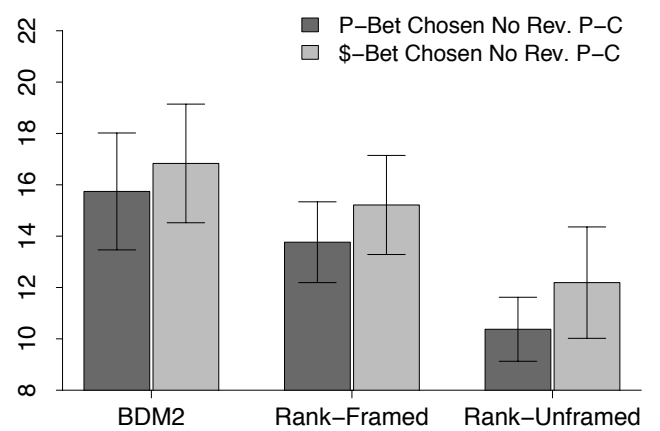

(a) Price/Rank-Choice

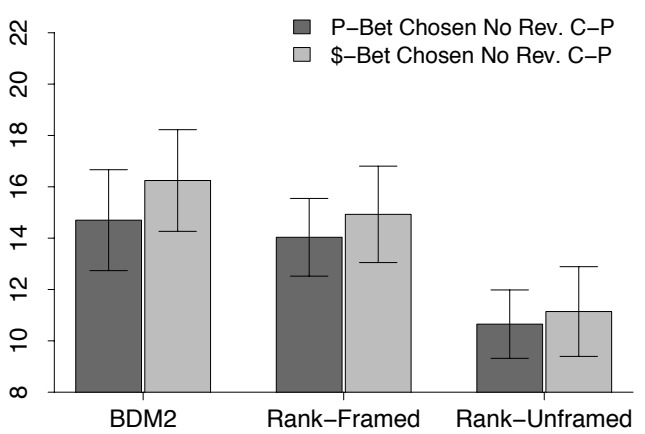

(b) Choice-Price/Rank

Figure 6: Average non-reversal decision time per individual in choice task of Experiment 2.

Note: Choices classified as non-reversals according to the indicated task ordering. Error bars depict the 95 percent confidence interval.

\subsection{Regression Analysis for Experiment 2}

As for Experiment 1, we conducted a random-effects panel regression analysis on the log of decision times from Experiment 2. The objective was to confirm and clarify our results while controlling for natural individual and lottery-pair characteristics; specifically, we included the same controls as in Experiment $1 .^{16}$ Table 2 contains the main results of all treatments. For each treatment, in the first regression reversals are classified as such according to the Price/RankChoice task ordering, while in the second one the Choice-Price/Rank is used. We present a single regression for each treatment and task ordering, but the results are robust with respect to the control variables.

Predicted reversals vs. non-reversals. The reversal dummies were highly significant in all treatments and task orderings, except for the "noisiest" Price-Choice in Treatment BDM2. This indicates that, as in Experiment 1, predicted reversals took longer than comparable nonreversals, confirming Hypothesis H2a.

Unpredicted reversals vs. non-reversals. Hypothesis $\mathrm{H} 2 \mathrm{~b}$ states that unpredicted reversals should take longer than non-reversals where the $\$$-bet was chosen. The difference corresponds to $\beta_{\text {Reversal }}+\beta_{\$-\text { Choice } \times \text { Reversal }}$, which is indeed highly significant and positive in all four regressions for the ranking treatments. The postestimation tests are not significant for the BDM2 treatment.

Comparison of non-reversals. The $\$$-choice dummy is significant and positive for Treatment BDM2. That is, as in Experiment 1, non-reversals where the \$-bet was chosen took longer than non-reversals where the $P$-bet was chosen in this treatment (Hypothesis H3). As stated in Hypothesis H7, we expected this effect to disappear for the purely ordinal, unframed treatment Rank-Unframed. Indeed, the dummy is not significant in any of the regressions for this treatment. The prediction is less clear for the "intermediate" treatment Rank-Framed, where

\footnotetext{
16 For the two ranking treatments, StatedDiff-1 and StatedDiff-3 refer to the difference in stated ranks between the two lotteries within a pair in phases 1 and 3 , respectively.
} 
Table 2: Random-effects panel regressions for decision times, Experiment 2.

\begin{tabular}{|c|c|c|c|c|c|c|}
\hline \multirow{2}{*}{$\begin{array}{l}\text { Treatment } \\
\text { Order }\end{array}$} & \multicolumn{2}{|c|}{ BDM2 } & \multicolumn{2}{|c|}{ RankFramed } & \multicolumn{2}{|c|}{ RankUnframed } \\
\hline & $\mathrm{P}-\mathrm{C}$ & C-P & $\mathrm{R}-\mathrm{C}$ & C-R & $\mathrm{R}-\mathrm{C}$ & C-R \\
\hline ReversalPC & $\begin{array}{r}0.049 \\
(0.031)\end{array}$ & & $\begin{array}{l}0.135^{\text {*** }} \\
(0.042)\end{array}$ & & $\begin{array}{l}0.108^{* * *} \\
(0.044)\end{array}$ & \\
\hline ReversalCP & & $\begin{array}{l}0.100^{* * *} \\
(0.032)\end{array}$ & & $\begin{array}{l}0.155^{* * *} \\
(0.043)\end{array}$ & & $\begin{array}{l}0.199^{* * *} \\
(0.051)\end{array}$ \\
\hline \$-Choice & $\begin{array}{c}0.072^{* *} \\
(0.033)\end{array}$ & $\begin{array}{l}0.098^{* * *} \\
(0.033)\end{array}$ & $\begin{array}{c}0.073^{* *} \\
(0.033)\end{array}$ & $\begin{array}{c}0.062^{*} \\
(0.033)\end{array}$ & $\begin{array}{r}0.030 \\
(0.036)\end{array}$ & $\begin{array}{r}-0.006 \\
(0.035)\end{array}$ \\
\hline $\begin{array}{l}\$ \text {-Choice } \\
\quad \times \text { ReversalPC }\end{array}$ & $\begin{array}{r}0.026 \\
(0.069)\end{array}$ & & $\begin{array}{r}0.014 \\
(0.062)\end{array}$ & & $\begin{array}{r}0.014 \\
(0.061)\end{array}$ & \\
\hline $\begin{array}{l}\text { \$-Choice } \\
\quad \times \text { ReversalCP }\end{array}$ & & $\begin{array}{r}0.011 \\
(0.077)\end{array}$ & & $\begin{array}{r}0.039 \\
(0.064)\end{array}$ & & $\begin{array}{r}0.008 \\
(0.067)\end{array}$ \\
\hline DiffEV & $\begin{array}{l}-0.022^{* *} \\
(0.031)\end{array}$ & $\begin{array}{l}-0.020^{*} \\
(0.011)\end{array}$ & $\begin{array}{l}-0.026^{* *} \\
(0.010)\end{array}$ & $\begin{array}{l}-0.025^{* *} \\
(0.010)\end{array}$ & $\begin{array}{r}-0.013 \\
(0.010)\end{array}$ & $\begin{array}{r}-0.008 \\
(0.010)\end{array}$ \\
\hline Ratio & $\begin{array}{l}0.034^{* * *} \\
(0.011)\end{array}$ & $\begin{array}{l}0.033^{* * *} \\
(0.011)\end{array}$ & $\begin{array}{l}0.038^{* * *} \\
(0.010)\end{array}$ & $\begin{array}{l}0.038^{* * *} \\
(0.010)\end{array}$ & $\begin{array}{l}0.044^{* * *} \\
(0.009)\end{array}$ & $\begin{array}{l}0.043^{* * *} \\
(0.009)\end{array}$ \\
\hline StatedDiff-1 & $\begin{array}{r}-0.005 \\
(0.005)\end{array}$ & $\begin{array}{r}-0.004 \\
(0.005)\end{array}$ & $\begin{array}{r}-0.011 \\
(0.010)\end{array}$ & $\begin{array}{l}-0.014 \\
(0.010)\end{array}$ & $\begin{array}{l}-0.024^{* *} \\
(0.010)\end{array}$ & $\begin{array}{l}-0.026^{* * *} \\
(0.010)\end{array}$ \\
\hline StatedDiff-3 & $\begin{array}{r}-0.003 \\
(0.005)\end{array}$ & $\begin{array}{l}-0.005 \\
(0.005)\end{array}$ & $\begin{array}{l}-0.046^{* * *} \\
(0.010)\end{array}$ & $\begin{array}{l}-0.041^{* * *} \\
(0.010)\end{array}$ & $\begin{array}{l}-0.045^{* * *} \\
(0.011)\end{array}$ & $\begin{array}{l}-0.042^{* * *} \\
(0.011)\end{array}$ \\
\hline Round & $\begin{array}{l}-0.007^{* * *} \\
(0.002)\end{array}$ & $\begin{array}{l}-0.007^{* * *} \\
(0.002)\end{array}$ & $\begin{array}{l}-0.008^{* * *} \\
(0.002)\end{array}$ & $\begin{array}{l}-0.007^{* * *} \\
(0.002)\end{array}$ & $\begin{array}{r}-0.003 \\
(0.002)\end{array}$ & $\begin{array}{r}-0.003 \\
(0.002)\end{array}$ \\
\hline Female & $\begin{array}{l}-0.190^{* *} \\
(0.089)\end{array}$ & $\begin{array}{l}-0.181^{* *} \\
(0.087)\end{array}$ & $\begin{array}{l}-0.238^{* * *} \\
(0.077)\end{array}$ & $\begin{array}{l}-0.237^{* * *} \\
(0.077)\end{array}$ & $\begin{array}{l}-0.217^{* *} \\
(0.087)\end{array}$ & $\begin{array}{l}-0.212^{* *} \\
(0.085)\end{array}$ \\
\hline Position & $\begin{array}{r}0.004 \\
(0.023)\end{array}$ & $\begin{array}{r}0.008 \\
(0.023)\end{array}$ & $\begin{array}{r}0.008 \\
(0.022)\end{array}$ & $\begin{array}{r}0.004 \\
(0.022)\end{array}$ & $\begin{array}{r}0.030 \\
(0.021)\end{array}$ & $\begin{array}{r}0.022 \\
(0.021)\end{array}$ \\
\hline Color & $\begin{array}{r}0.108 \\
(0.087)\end{array}$ & $\begin{array}{r}0.112 \\
(0.086)\end{array}$ & $\begin{array}{r}0.088 \\
(0.077)\end{array}$ & $\begin{array}{r}0.085 \\
(0.077)\end{array}$ & $\begin{array}{r}-0.034 \\
(0.085)\end{array}$ & $\begin{array}{l}-0.040 \\
(0.083)\end{array}$ \\
\hline Constant & $\begin{array}{c}2.624^{* * *} \\
(0.095)\end{array}$ & $\begin{array}{c}2.595^{\text {*** }} \\
(0.094)\end{array}$ & $\begin{array}{c}2.649^{* * *} \\
(0.087)\end{array}$ & $\begin{array}{r}2.645 \\
(0.087) \\
\end{array}$ & $\begin{array}{c}2.391^{* * *} \\
(0.090)\end{array}$ & $\begin{array}{c}2.388^{* * *} \\
(0.088)\end{array}$ \\
\hline Nr. Obs. & 1242 & 1244 & 1314 & 1314 & 1314 & 1314 \\
\hline Nr. Groups & 69 & 69 & 73 & 73 & 73 & 73 \\
\hline R2-Overall & 0.066 & 0.069 & 0.128 & 0.140 & 0.104 & 0.122 \\
\hline Wald test & 0.000 & 0.000 & 0.000 & 0.000 & 0.000 & 0.000 \\
\hline Postestimation & tests & & & & & \\
\hline $\begin{array}{l}\text { Reversal } \\
\quad+(\$ \text {-Ch } \times \text { Rev })\end{array}$ & $\begin{array}{r}0.075 \\
(0.060)\end{array}$ & $\begin{array}{r}0.111 \\
(0.068)\end{array}$ & $\begin{array}{l}0.149^{* * *} \\
(0.043)\end{array}$ & $\begin{array}{l}0.194^{* * *} \\
(0.044)\end{array}$ & $\begin{array}{l}0.121^{* * *} \\
(0.031)\end{array}$ & $\begin{array}{l}0.207^{* * *} \\
(0.041)\end{array}$ \\
\hline
\end{tabular}

Note: All regressions are random-effects panel estimations, with log decision time as dependent variable. Standard errors in parentheses. ${ }^{* * *} p<0.01,{ }^{* *} p<0.05,{ }^{*} p<0.1$. 
the evaluation task was also ordinal but there was an indirect framing in terms of prices. ${ }^{17}$ For this treatment, the $\$$-choice was significantly positive, but e.g. only at the $10 \%$ level for the Choice-Rank ordering.

Controls: Lotteries. As in Experiment 1, the ratio of the two higher amounts to win in the $\$$-bet and the $P$-bet (Ratio) had a significant positive effect throughout. Likewise, the absolute difference in expected values of the $P$-bet and the $\$$-bet (DiffEV) had a weakly significant negative effect, but not in the Rank-Unframed treatment. The absolute difference in the prices/ranks stated for the lotteries in phases one and three (StatedDiff-1, StatedDiff-3) was significant for Rank-Unframed but not for BDM2 (and only the second measure was significant for Rank-Framed).

Other controls. As in Experiment 1, we controlled for learning and familiarity effects by including the round in which the choice was made as a regressor. Also as in Experiment 1, female participants were significantly quicker in all treatments and task orderings. The onscreen position (Position) of the $P$-bet and the $\$$-bet and the colors used in the pie-chart (Color) had, as expected, no effect.

\subsection{Discussion of Experiment 2}

The experiment successfully separated the two postulated determinants of reversals. On the one hand, the almost-complete disappearance of predicted reversals in the ranking treatments (especially when ordinally framed) confirms that the overpricing phenomenon can be shut down. It also shows that this phenomenon appears due to the cardinal, rating-based frame used in standard evaluation tasks as those employed in Experiment 1. On the other hand, the remaining reversals are still associated with longer decision times (a prediction we derived from noisy evaluations) even though the overpricing process has been impaired. This is evidence that both noisy evaluations and the overpricing phenomenon need to be taken into account as different ingredients in order to model preference reversals.

Concerning the elimination of reversals, our results are consistent with evidence from Bateman et al. (2007). These authors also observed a reduction in predicted preference reversal rates in an experiment where lotteries were ranked within sets which also contained sure amounts. Their ranking task is not directly comparable to ours because $\$$-bets and $P$-bets were ranked separately, i.e. within different sets, and the ranks of $P$-bets relative to $\$$-bets were inferred indirectly. Oliver (2013) used a similar method for the measurement of preferences in the health domain (life expectancy).

Our main object of study have been predicted preference reversals, since they are empirically more relevant. The compatibility hypothesis points to an overpricing phenomenon as a reason for the predominance of these reversals, while the origin of unpredicted ones might be just noisy evaluations. Nonetheless, it is interesting to observe that the rate of unpredicted reversals increased in the ranking treatments with respect to the control (BDM2) treatment. In fact, our ranking treatments reversed the usual pattern of preference reversals turning un-

\footnotetext{
17 We consider the framing "indirect" because, contrary to the tasks in Experiment 1 or Treatment BDM2, participants did not actually write down prices.
} 
predicted reversals into the dominant ones. We hypothesize that, when the cues on which the overpricing process acts are removed, attention is diverted to probabilities instead. Following the compatibility hypothesis, this would result in an over-evaluation of $P$-bets, for which a high probability is salient. However, this process is weaker than the one causing overpricing of $\$$ bets with pricing frames, simply because monetary rewards are a more immediately accessible concept than probabilities. Thus, in a standard preference-reversal study, this second process is overshadowed by the overpricing of $\$$-bets. Our evidence in this respect is consistent with Cubitt et al. (2004), where the rate of unpredicted reversals increased when subjects were asked for "probabilistic valuations" instead of prices, trying to induce a probability anchor. However, the rates of predicted reversals remained relatively high, suggesting that such valuation tasks, being still cardinal, do not completely remove the salience of monetary outcomes. ${ }^{18}$ Casey (1991, 1994) observed a higher rate of unpredicted reversals compared to predicted ones using very high payoffs and maximum buying prices (rather than minimum selling prices). Again, however, predicted reversal rates remained comparatively high. Casey (1994) argues that high stakes might induce buyers to anchor on the smallest monetary outcome of a lottery, adjusting the valuation upwards on the basis of probabilities, and hence resulting in an overpricing of P-bets. In our terms, the setting of Casey $(1991,1994)$ might correspond to a combination of elements enhancing the second process mentioned above. If such a second process is assumed, the increase of unpredicted reversals in our ranking treatments, in Cubitt et al. (2004), and in Casey (1991, 1994) can be explained within our model. One could argue that in a ranking task there is, for whatever the reason, a tendency to "over-rank" the P-bet. Versions of our Propositions 1 and 2 would then yield a higher rate of unpredicted reversals as well as the observed decision time pattern for reversals. This, however, would not explain the fact that $\$$-bet non-reversal choices still take (weakly) longer that $P$-bet non-reversal choices in the ranking treatments. The latter observation might point towards a possible "over-ranking" of $P$-bets being guided by a fundamentally different process than the overpricing of $\$$-bets.

As a remark, we observe order effects on decision times (similar to those already seen in Experiment 1) that are compatible with the view that post-choice elicitation tasks carry less noise than pre-choice analogues, possibly due to "preference sharpening" or reappraisal in the sense of self-perception theory.

\subsection{Why Are Choices After Rankings Quicker? A Dual-Process Explanation}

An important observation is that decision times in Treatment Rank-Unframed were significantly shorter than those in other treatments, with the difference being also rather large (see Figures 5 and 6). This is striking, because the choice phase in all our treatments was identical. Actually, this difference was expected, and the reasons have to be sought in (psychological) theories of decision processes. ${ }^{19}$

\footnotetext{
18 Participants were asked for the probability $p$ making them indifferent between a given lottery and receiving a fixed, high monetary outcome $X$ with probability $p$. Hence monetary outcomes remained an important part of the frame.

19 We present this discussion after the fact for readability reasons only. For a detailed discussion of decision times and conflicting decision processes under risk and uncertainty, see Achtziger and Alós-Ferrer (2014).
} 
We focus on dual-process models from psychology (Strack and Deutsch, 2004; Evans, 2008; Weber and Johnson, 2009; Alós-Ferrer and Strack, 2014), which postulate that decisions are the result of the interaction of possibly conflicting decision processes of different types. A basic prediction of dual-process models is that the detection of conflict among decision processes and its resolution consume both time and cognitive resources. That is, decision times are longer when several processes conflict than when processes are aligned. In particular, this is independent of how the conflict is resolved, i.e. which decision is actually made and which process determines this decision.

In our framework, the compatibility hypothesis suggests that several decision processes might be at work when choosing from a pair of lotteries. Overpricing might result from a process focusing on monetary outcomes only, which competes with a more global decision process that evaluates lotteries by taking both outcomes and winning probabilities into account. Clearly, the former process is activated by a monetary frame in the initial evaluation phase (pricing) and will be especially prevalent during lottery evaluation. If activated, this decision process remains active during the subsequent choice phase, but there it is often inhibited, which leads to a choice causing a preference reversal. The resulting process conflict results in increased decision times. In the Treatment Rank-Unframed, we removed all references to prices, and hence the overpricing process was simply not activated at all. Hence, there was no process conflict, and no additional time was spent in conflict resolution in the choice phase. It is interesting to observe that in the Treatment Rank-Framed, where the evaluation task was also ordinal but the frame made a reference to prices, decision times were closer to those of the rating treatments, even though predicted reversals were also greatly reduced. Again, the interpretation is simple. The price frame generally activated the process behind overpricing, but the fact that the task was ultimately a purely ordinal one made it less likely that this process actually shaped the decision in the evaluation tasks, hence reducing reversals. However, since the process had been activated, it needed to be inhibited in the choice phase, causing longer decision times.

This explanation does not affect our other predictions. Essentially, it means that decision times in the choice task, $D T$, consist of two components, choice time $D T_{C}$ and conflict resolution time $D T_{R}$, i.e. $D T=D T_{C}+D T_{R}$, where $D T_{R}$ is larger in the presence of process conflict. Within each experimental treatment, there are no large variations in $D T_{R}$, and hence none of the predictions are affected.

Two comments are in order. First, it is conceivable that some non-reversals correspond to cases where the overpricing process is not activated. This would lead to the additional assumption that, within a treatment, conflict resolution time is larger for reversals than for comparable non-reversals. ${ }^{20}$ This more detailed account would leave our other hypotheses unaffected, because H1/H5 do not concern decision times, and do not concern reversals. Hypothesis H4 on order effects is likewise orthogonal to this question. The additional assumption would affect exclusively the interpretation of Hypotheses $\mathrm{H} 2 / \mathrm{H} 7$, i.e. that reversals take longer than comparable nonreversals. However, Proposition 2 states that choice times are longer for reversals

\footnotetext{
20 Such an assumption would also capture the observation that preference reappraisal and process conflict resolution in the Free-Choice Paradigm occur during the choice phase, as observed in fMRI (Jarcho et al., 2011) and response time studies (Alós-Ferrer et al., 2012).
} 
than for non-reversals. The new assumption would state that also conflict resolution time is on average larger for reversals. Since total decision time is now viewed as the sum of choice time and conflict resolution time, Hypotheses H2/H7 would remain unaffected.

Second, dual-process theories postulate that decision processes can range from automatic and fast, corresponding to quick heuristics, to controlled and slow. Although inessential for our analysis, it is interesting to speculate on the nature of the decision processes involved in preference reversals. Clearly, a decision process reflecting global valuation considerations should be conceptualized as a cognitive, controlled process. It would be reasonable to assume that the overpricing process is more automatic (quicker) than the former process. Our results suggest, however, that it cannot be a purely impulsive process with very low response times. If this were the case, then in case of conflict some decisions would actually be made by the impulsive process and be correspondingly far quicker. This would reduce the average decision time, operating in the opposite direction to the effect discussed above. Our data make clear that this process is not quick enough for this second-order consideration to offset the reduction of conflict resolution time.

\section{Conclusion}

We investigated decision times in the choice task of two preference reversal experiments to deepen our understanding of the actual decision processes behind preference reversals. Our main hypotheses separate the reasons for the existence of reversals and for the predominance of predicted reversals on the basis of two prominent accounts from the literature. While the existence of reversals is attributed to noise (imprecise preferences) in the evaluation phase (Schmidt and Hey, 2004; Butler and Loomes, 2007), the predominance of predicted reversals is the result of an overpricing phenomenon of lotteries with salient monetary outcomes associated with the compatibility hypothesis (Tversky et al., 1988, 1990). A noisy evaluation phase suggests that choices involved in preference reversals are more likely to include comparisons where decision makers are closer to indifference. Since decision times associated with such hard choices have been shown to take longer than easy choices where one option is clearly better (Wilcox, 1993; Shultz et al., 1999; Moffatt, 2005; Chabris et al., 2009; Sharot et al., 2009; Alós-Ferrer et al., 2012), choices involved in preferences reversals should take longer than corresponding non-reversal choices.

The first experiment confirmed these hypotheses. We reproduced the standard preference reversal pattern and found that reversals were clearly associated with longer decision times. In the second experiment, we were able to shut down the overpricing process by moving away from cardinal-based evaluation phases to purely ordinal ranking tasks, which effectively separates the consequences of the two postulated determinants of reversals. As expected, we observe a significant reduction of predicted reversals. More importantly, reversal choices still take longer than non-reversal choices, a prediction derived from imprecise preferences only. These findings are compatible with the idea that reversals arise due to an interaction of noise in the evaluation phases and a psychological process (or set thereof) causing an overpricing of lotteries with a 
salient monetary outcome.

In addition, we found that a complete removal of cues to prices in the evaluation phase notably reduces decision times in the choice task. This observation is remarkable insofar as we employ two otherwise identical ranking tasks that only differ in the experimental instructions. In one treatment (Rank-Framed) participants are instructed to think about minimum selling prices and rank lotteries accordingly, in the other (Rank-Unframed) there is no mentioning of prices at all. Thinking about minimum selling prices in the evaluation phase significantly increases decision times in the choice phase. This finding is compatible with standard psychological insights on process conflict and provides further support towards the existence of an overpricing process triggered by price cues.

Our design further allowed us to evaluate different experimental possibilities with regard to the amount of noise they induce. By using two evaluation phases, one pre-choice and one postchoice, we are able to conclude that post-choice evaluation tasks are in general more appropriate for preference elicitation, in accordance with evidence on preference reappraisal from psychology. By using different evaluation tasks across treatments, we conclude that tasks based on the BDM procedure might add additional, unwanted noise and other tasks, as e.g. the Ordinal Payment Method, might be more accurate. Finally, if one is interested in preferences rather than certainty equivalents, our second experiment shows that the more accurate evaluation method (in the sense of inducing fewer reversals) is to rely on purely ordinal, ranking-based tasks without any references to prices.

Our research investigated the mechanisms and processes behind the preference reversal phenomenon. Previous research (see e.g. Cubitt et al., 2004) has pointed out that a combination of psychological mechanisms might be the simplest explanation of the phenomenon. Given the fundamental importance of preference elicitation methods for both decision theory and applied economics, and the amount of attention dedicated to the preference reversal phenomenon in the last half century, we believe that fleshing out these mechanisms is an important step. At the same time, we show that a parsimonious combination of insights from the literature with standard facts on decision times can account for received evidence and provide new, testable hypotheses allowing us to better understand the determinants of the preference reversal phenomenon.

\section{References}

Achtziger, Anja and Carlos Alós-Ferrer (2014), "Fast or rational? A response-times study of Bayesian updating." Management Science, 60, 923-938.

Alós-Ferrer, Carlos, Dura-Georg Granić, Fei Shi, and Alexander K. Wagner (2012), "Choices and preferences: Evidence from implicit choices and response times." Journal of Experimental Social Psychology, 48, 1336-1342.

Alós-Ferrer, Carlos and Fei Shi (2015), "Spreading might be informative in the free-choice paradigm." Judgment and Decision Making, 10, 34-49. 
Alós-Ferrer, Carlos and Fritz Strack (2014), "From dual processes to multiple selves: Implications for economic behavior." Journal of Economic Psychology, 41, 1-11.

Bargh, John A. and Tanya L. Chartrand (2000), "The Mind in the Middle: A Practical Guide to Priming and Automaticity Research." In Handbook of Research Methods in Social and Personality Psychology (Harry T. Reis and Charles M. Judd, eds.), 253-285, Cambridge University Press, NY.

Bateman, Ian, Brett Day, Graham Loomes, and Robert Sugden (2007), "Can ranking techniques elicit robust values?" Journal of Risk and Uncertainty, 34, 49-66.

Becker, Gordon M., Morris H. DeGroot, and Jacob Marschak (1964), "Measuring utility by a single response sequential method." Behavioral Science, 9, 226-232.

Bem, Daryl (1967), "Self-perception: An alternative interpretation of cognitive dissonance phenomena." Psychological Review, 74, 183-200.

Blavatsky, Pavlo R. (2009), "Preference reversals and probabilistic decisions." Journal of Risk and Uncertainty, 39, 237-250.

Bleichrodt, Han and José Luis Pinto Prades (1994), "New evidence of preference reversals in health utility measurement." Health Economics, 18, 713-726.

Brehm, Jack W. (1956), "Postdecision changes in the desirability of alternatives." Journal of Abnormal and Social Psychology, 52, 384-389.

Butler, David J. and Graham Loomes (2007), "Imprecision as an account of the preference reversal phenomenon." The American Economic Review, 97, 277-297.

Casey, Jeff T. (1991), "Reversal of the preference reversal phenomenon." Organizational Behavior and Human Decision Processes, 48, 224-251.

Casey, Jeff T. (1994), "Buyers' pricing behavior for risky alternatives: Encoding processes and preference reversals." Management Science, 40, 730-749.

Chabris, Christopher F., David I. Laibson, Carrie L. Morris, Jonathon P. Schuldt, and Dmitry Taubinsky (2009), "The allocation of time in decision-making." Journal of the European Economic Association, 7, 682-637.

Cubitt, Robin P., Alistair Munro, and Chris Starmer (2004), "Testing explanations of preference reversal." The Economic Journal, 114, 709-726.

Delquié, Philippe (1993), "Inconsistent trade-offs between attributes: New evidence in preference assessment biases." Management Science, 39, 1382-1395.

Evans, Jonathan St. B. T. (2008), "Dual-Processing Accounts of Reasoning, Judgment, and Social Cognition." Annual Review of Psychology, 59, 255-278.

Festinger, Leon (1957), A Theory of Cognitive Dissonance. Stanford University Press, Stanford. 
Fischbacher, Urs (2007), "z-Tree: Zurich toolbox for ready-made economic experiments." Experimental Economics, 10, 171-178.

Fischer, Gregory W., Ziv Carmon, Dan Ariely, and Gal Zauberman (1999), "Goal-based construction of preferences: Task goals and the prominence effect." Management Science, 45, $1057-1075$.

Goldstein, William M. and Hillel J. Einhorn (1987), "Expression theory and the preference reversal phenomena." Psychological Review, 94, 236-254.

Grether, David M. and Charles R. Plott (1979), "Theory of choice and the preference reversal phenomenon." The American Economic Review, 69, 623-638.

Hershey, John C. and Paul J. H. Schoemaker (1985), "Probability versus certainty equivalence methods in utility measurement: Are they equivalent?" Management Science, 31, 1213-1231.

Hey, John D. (2001), "Does Repetition Improve Consistency?" Experimental Economics, 4, $5-54$.

Jarcho, Johanna M., Elliot T. Berkman, and Matthew D. Lieberman (2011), "The neural basis of rationalization: Cognitive dissonance reduction during decision-making." Social Cognitive and Affective Neuroscience, 6, 460-467.

Johnson, Eric J. and David A. Schkade (1989), "Bias in utility assessments: Further evidence and explanations." Management Science, 35, 406-424.

Lindman, Harold R. (1971), "Inconsistent preferences among gambles." Journal of Experimental Psychology, 89, 390-397.

Lindsay, Luke (2013), "The arguments of utility: Preference reversals in expected utility of income models." Journal of Risk and Uncertainty, 46, 175-189.

Moffatt, Peter G. (2005), "Stochastic Choice and the Allocation of Cognitive Effort." Experimental Economics, 8, 369-388.

Oliver, Adam (2013), "Testing procedural invariance in the context of health." Health Economics, 22, 272-288.

Rubinstein, Ariel (2007), "Instinctive and Cognitive Reasoning: A Study of Response Times." Economic Journal, 117, 1243-1259.

Schkade, David A. and Eric J. Johnson (1989), "Cognitive processes in preference reversals." Organizational Behavior and Human Decision Processes, 44, 203-231.

Schmidt, Ulrich and John D. Hey (2004), "Are preference reversals errors? An experimental investigation." The Journal of Risk and Uncertainty, 29, 207-218.

Schmidt, Ulrich, Chris Starmer, and Robert Sugden (2008), "Third-generation prospect theory." Journal of risk and uncertainty, 36, 203-223. 
Seidl, Christian (2002), "Preference reversal." Journal of Economic Surveys, 16, 621-655.

Sharot, Tali, Benedetto De-Martino, and Raymond J. Dolan (2009), "How Choice Reveals and Shapes Expected Hedonic Outcome." The Journal of Neuroscience, 29, 3760-3765.

Shultz, Thomas R., Elé Léveillé, and Mark R. Lepper (1999), "Free choice and cognitive dissonance revisited: Choosing "lesser evils" versus "greater goods"." Personality and Social Psychology Bulletin, 25, 40-48.

Slovic, Paul and Sarah Lichtenstein (1968), "Relative importance of probabilities and payoffs in risk taking." Journal of Experimental Psychology Monograph, 78, 1-18.

Stalmeier, Peep F. M., Peter P. Wakker, and Thom G. G. Bezembinder (1997), "Preference reversals: Violations of unidimensional procedure invariance." Journal of Experimental Psychology: Human Perception and Performance, 23, 1196-1205.

Strack, Fritz and Roland Deutsch (2004), "Reflective and Impulsive Determinants of Social Behavior." Personality and Social Psychology Review, 8, 220-247.

Sugden, Robert (2003), "Reference-dependent subjective expected utility." Journal of Economic Theory, 111, 172-191.

Tversky, Amos, Shmuel Sattath, and Paul Slovic (1988), "Contingent weighting in judgment and choice." Psychological Review, 95, 371-384.

Tversky, Amos, Paul Slovic, and Daniel Kahneman (1990), "The causes of preference reversal." The American Economic Review, 80, 204-217.

Weber, Elke U. and Eric J. Johnson (2009), "Mindful Judgment and Decision Making." Annual Review of Psychology, 60, 53-85.

Wilcox, Nathaniel T. (1993), "Lottery Choice: Incentives, Complexity, and Decision Time." Economic Journal, 103, 1397-1417.

Wilcox, Nathaniel T. (1994), "On a Lottery Pricing Anomaly: Time Tells the Tale." Journal of Risk and Uncertainty, 8, 311-324. 


\title{
Supplementary Online Material for Preference Reversals: Time and Again
}

\author{
Carlos Alós-Ferrer ${ }^{a} \quad$ Dura-Georg Granić ${ }^{b} \quad$ Johannes Kern $^{c}$ \\ Alexander K. Wagner ${ }^{d}$
}

This version: August, 2015

The supplementary online material contains the proofs of analytical results, the table of lotteries used in the experiments, and screenshots.

\footnotetext{
${ }^{a}$ Department of Economics, University of Cologne, Albertus-Magnus Platz, D-50923 Cologne, Germany. Email: carlos.alos-ferrer@uni-koeln.de

${ }^{b}$ Department of Economics, University of Cologne, Albertus-Magnus Platz, D-50923 Cologne, Germany. Email: georg.granic@uni-koeln.de

${ }^{c}$ Department of Economics, University of Cologne, Albertus-Magnus Platz, D-50923 Cologne, Germany. Email: johannes.kern@uni-koeln.de

${ }^{d}$ Department of Economics, University of Cologne, Albertus-Magnus Platz, D-50923 Cologne, Germany. Present Address: Vienna Center for Experimental Economics and Department of Economics, University of Vienna, Oskar-Morgenstern-Platz 1, A-1090 Vienna, Austria. Email: alexander.k.wagner@univie.ac.at
} 


\section{A Proofs}

Throughout the appendix, let $\Delta \zeta=\zeta_{P}-\zeta_{\$}+K$. Under Assumption 3, $\zeta_{P}$ and $\zeta_{\$}$ are i.i.d. and unimodal, implying that $\Delta \zeta$ is symmetrically distributed around 0 and unimodal (cf. Purkayastha, 1998, Theorem 2.1).

Proof of Proposition 1. We first claim that the probability of a predicted reversal $\operatorname{Pr}\left(C E_{\$}>\right.$ $\left.C E_{P}, C(P, \$)=P\right)$ is higher than the probability of an unpredicted reversal $\operatorname{Pr}\left(C E_{P}>\right.$ $\left.C E_{\$}, C(P, \$)=\$\right)$. Since $K>0$ by Assumption $3, \operatorname{Pr}(\Delta \zeta<-K-s)<\operatorname{Pr}(\Delta \zeta<K-s)$ for all $s \in[0, \infty[$ and the conclusion follows from the following computations.

$$
\begin{aligned}
\operatorname{Pr}\left(C E_{\$}>C E_{P}, C(P, \$)=P\right) & \int_{0}^{\infty} \operatorname{Pr}\left(C E_{\$}>C E_{P} \mid u_{P}-u_{\$}=s\right) h(s) d s=\int_{0}^{\infty} \operatorname{Pr}(\Delta \zeta<K-s) h(s) d s \\
\operatorname{Pr}\left(C E_{P}>C E_{\$}, C(P, \$)=\$\right) & =\int_{0}^{\infty} \operatorname{Pr}\left(C E_{P}>C E_{\$} \mid u_{\$}-u_{P}=s\right) h(s) d s \\
& =\int_{0}^{\infty} \operatorname{Pr}(\Delta \zeta>K+s) h(s) d s=\int_{0}^{\infty} \operatorname{Pr}(\Delta \zeta<-K-s) h(s) d s .
\end{aligned}
$$

This proves the claim.

Note that $\operatorname{Pr}\left(C E_{\$}>C E_{P} \mid c(P, \$)=P\right)=\frac{\operatorname{Pr}\left(C E_{\$}>C E_{P}, c(P, \$)=P\right)}{\operatorname{Pr}\left(u_{P}>u_{\S}\right)}$, and $\operatorname{Pr}\left(C E_{P}>C E_{\$} \mid c(P, \$)=\right.$ $\$)=\frac{\operatorname{Pr}\left(C E_{P}>C E_{\S}, c(P, \$)=\$\right)}{\operatorname{Pr}\left(u_{\S}>u_{P}\right)}$. Since $\operatorname{Pr}\left(u_{P}>u_{\$}\right)=\operatorname{Pr}\left(u_{\$}>u_{P}\right)$, the conclusion follows from the claim.

The next lemma is used in the proof of Proposition 2.

Lemma 1. Under Assumption 1, the following hold.

(i) $\operatorname{Pr}\left(C E_{\$}>C E_{P} \mid 0<u_{P}-u_{\$}<\delta\right)>\operatorname{Pr}\left(C E_{\$}>C E_{P} \mid u_{P}-u_{\$}>\delta\right)$.

(ii) $\operatorname{Pr}\left(C E_{P}>C E_{\$} \mid 0<u_{\$}-u_{P}<\delta\right)>\operatorname{Pr}\left(C E_{P}>C E_{\$} \mid u_{\$}-u_{P}>\delta\right)$.

Proof. We prove part (i). The proof of part (ii) is analogous. We have $\operatorname{Pr}\left(C E_{\$}>C E_{P} \mid u_{P}-u_{\$}=\right.$ $s)=\operatorname{Pr}(\Delta \zeta<K-s)$ and

$$
\begin{aligned}
\operatorname{Pr}\left(C E_{\$}>C E_{P} \mid 0\right. & \left.<u_{P}-u_{\$}<\delta\right)=\frac{1}{\operatorname{Pr}\left(0<u_{P}-u_{\$}<\delta\right)} \int_{0}^{\delta} \operatorname{Pr}\left(C E_{\$}>C E_{P} \mid u_{P}-u_{\$}=s\right) h(s) d s \\
& >\frac{1}{\operatorname{Pr}\left(0<u_{P}-u_{\$}<\delta\right)} \int_{0}^{\delta} \operatorname{Pr}(\Delta \zeta<K-\delta) h(s) d s=\operatorname{Pr}(\Delta \zeta<K-\delta) .
\end{aligned}
$$

Similarly $\operatorname{Pr}\left(C E_{\$}>C E_{P} \mid u_{P}-u_{\$}=s\right)=\operatorname{Pr}(\Delta \zeta<K-s)$ and

$$
\begin{array}{r}
\operatorname{Pr}\left(C E_{\$}>C E_{P} \mid u_{P}-u_{\$}>\delta\right)=\frac{1}{\operatorname{Pr}\left(u_{P}-u_{\$}>\delta\right)} \int_{\delta}^{\infty} \operatorname{Pr}\left(C E_{\$}>C E_{P} \mid u_{P}-u_{\$}=s\right) h(s) d s \\
<\frac{1}{\operatorname{Pr}\left(u_{P}-u_{\$}>\delta\right)} \int_{\delta}^{\infty} \operatorname{Pr}(\Delta \zeta<K-\delta) h(s) d s=\operatorname{Pr}(\Delta \zeta<K-\delta)
\end{array}
$$

and the conclusion follows. 
Proof of Proposition 2. (i) To shorten notation let $\Delta_{0}=\operatorname{Pr}\left(C E_{\$}>C E_{P} \mid 0<u_{P}-u_{\$}<\delta\right)$, $\Delta_{1}=\operatorname{Pr}\left(C E_{\$}>C E_{P} \mid u_{P}-u_{\$}>\delta\right), P^{\delta}=\operatorname{Pr}\left(0<u_{P}-u_{\$}<\delta \mid 0<u_{P}-u_{\$}\right)$, and $P=$ $\operatorname{Pr}\left(C E_{\$}>C E_{P} \mid u_{P}>u_{\$}\right)$.

With these definitions, $P=\Delta_{0} P^{\delta}+\Delta_{1}\left(1-P^{\delta}\right)$. We obtain $E\left[D T_{C} \mid C E_{\$}>C E_{P}, c(P, \$)=\right.$ $P]=\frac{1}{P}\left[\Delta_{0} P^{\delta} T_{H}+\Delta_{1}\left(1-P^{\delta}\right) T_{E}\right]$, and $E\left[D T_{C} \mid C E_{P}>C E_{\$}, c(P, \$)=P\right]=\frac{1}{1-P}\left[\left(1-\Delta_{0}\right) P^{\delta} T_{H}+\right.$ $\left.\left(1-\Delta_{1}\right)\left(1-P^{\delta}\right) T_{E}\right]$. A simple calculation shows that

$$
\begin{aligned}
E\left[D T_{C} \mid C E_{\$}>C E_{P}, c(P, \$)=P\right]>E\left[D T_{C} \mid C E_{P}\right. & \left.>C E_{\$}, c(P, \$)=P\right] \\
& \Leftrightarrow P^{\delta} T_{H}\left[\Delta_{0}-P\right]>\left(1-P^{\delta}\right) T_{E}\left[P-\Delta_{1}\right]
\end{aligned}
$$

As $P=\Delta_{0} P^{\delta}+\Delta_{1}\left(1-P^{\delta}\right)$, we obtain $\Delta_{0}-P=\left(1-P^{\delta}\right)\left(\Delta_{0}-\Delta_{1}\right)$ and $P-\Delta_{1}=P^{\delta}\left(\Delta_{0}-\Delta_{1}\right)$. Hence $E\left[D T_{C} \mid C E_{\$}>C E_{P}, c(P, \$)=P\right]>E\left[D T_{C} \mid C E_{P}>C E_{\$}, c(P, \$)=P\right]$ holds if and only if $T_{H}\left(\Delta_{0}-\Delta_{1}\right)>T_{E}\left(\Delta_{0}-\Delta_{1}\right)$. By Lemma 1(i), $\Delta_{0}>\Delta_{1}$ and hence the inequality holds if and only if $T_{H}>T_{E}$, which is true by Assumption 4 .

(ii) is analogous to (i), using part (ii) of Lemma 1 instead of (i).

The next lemma is used in the proof of Proposition 3.

Lemma 2. $\operatorname{Pr}\left(0<u_{P}-u_{\$}<\delta \mid 0<u_{P}-u_{\$}\right)=\operatorname{Pr}\left(0<u_{\$}-u_{P}<\delta \mid 0<u_{\$}-u_{P}\right)$.

Proof. First note that since $u_{P}$ and $u_{\$}$ are i.i.d, $u_{P}-u_{\$}$ and $u_{\$}-u_{P}$ are identically distributed and $\operatorname{Pr}\left(u_{\$}-u_{P}>0\right)=\operatorname{Pr}\left(u_{\$}-u_{P}<0\right)=1 / 2$. Then $\operatorname{Pr}\left(0<u_{\$}-u_{P}<\delta \mid 0<u_{\$}-u_{P}\right)=$ $\frac{\operatorname{Pr}\left(0<u_{\S}-u_{P}<\delta\right)}{\operatorname{Pr}\left(u_{\S}>u_{P}\right)}=\frac{\operatorname{Pr}\left(0<u_{P}-u_{\S}<\delta\right)}{\operatorname{Pr}\left(u_{P}>u_{\S}\right)}=\operatorname{Pr}\left(0<u_{P}-u_{\$}<\delta \mid 0<u_{P}-u_{\$}\right)$.

Proof of Proposition 3. To shorten notation let $\Delta_{0}=\operatorname{Pr}\left(C E_{\$}>C E_{P} \mid 0<u_{\$}-u_{P}<\delta\right)$, $\Delta_{1}=\operatorname{Pr}\left(C E_{\$}>C E_{P} \mid u_{\$}-u_{P}>\delta\right), \Delta_{2}=\operatorname{Pr}\left(C E_{P}>C E_{\$} \mid 0<u_{P}-u_{\$}<\delta\right), \Delta_{3}=\operatorname{Pr}\left(C E_{P}>\right.$ $\left.C E_{\$} \mid u_{P}-u_{\$}>\delta\right), P_{1}=\operatorname{Pr}\left(C E_{\$}>C E_{P} \mid u_{\$}>u_{P}\right), P_{2}=\operatorname{Pr}\left(C E_{P}>C E_{\$} \mid u_{P}>u_{\$}\right)$. Let also $P^{\delta}$ be the probability given in Lemma 2 .

With these definitions, we have that $P_{1}=\Delta_{0} P^{\delta}+\Delta_{1}\left(1-P^{\delta}\right)$ and $P_{2}=\Delta_{2} P^{\delta}+\Delta_{3}\left(1-P^{\delta}\right)$.

We obtain $E\left[D T_{C} \mid C E_{\$}>C E_{P}, c(P, \$)=\$\right]=\frac{1}{P_{1}}\left[\Delta_{0} P^{\delta} T_{H}+\Delta_{1}\left(1-P^{\delta}\right) T_{E}\right]$ and $E\left[D T_{C} \mid C E_{P}>\right.$ $\left.C E_{\$}, c(P, \$)=P\right]=\frac{1}{P_{2}}\left[\Delta_{2} P^{\delta} T_{H}+\Delta_{3}\left(1-P^{\delta}\right) T_{E}\right]$. This yields.

$$
\begin{aligned}
E\left[D T_{C} \mid C E_{\$}>C E_{P}, c(P, \$)\right. & =\$]>E\left[D T_{C} \mid C E_{P}>C E_{\$}, c(P, \$)=P\right] \\
\Leftrightarrow & P^{\delta}\left(1-P^{\delta}\right) T_{H}\left[\Delta_{0} \Delta_{3}-\Delta_{1} \Delta_{2}\right]>\left(1-P^{\delta}\right) P^{\delta} T_{E}\left[\Delta_{0} \Delta_{3}-\Delta_{1} \Delta_{2}\right]
\end{aligned}
$$

Since $T_{H}>T_{E}$ by Assumption 4, the claim holds if $\Delta_{1} \Delta_{2}<\Delta_{0} \Delta_{3}$. The rest of the proof is devoted to establish this fact. For this, we rely on ideas taken from Wijsman (1985).

First, note that

$$
\begin{aligned}
& \Delta_{0}=\frac{1}{\operatorname{Pr}\left(0<u_{\$}-u_{P}<\delta\right)} \int_{0}^{\delta} \operatorname{Pr}(\Delta \zeta<K+s) h(s) d s, \\
& \Delta_{1}=\frac{1}{\operatorname{Pr}\left(u_{\$}-u_{P}>\delta\right)} \int_{\delta}^{\infty} \operatorname{Pr}(\Delta \zeta<K+s) h(s) d s, \\
& \Delta_{2}=\frac{1}{\operatorname{Pr}\left(0<u_{p}-u_{\$}<\delta\right)} \int_{0}^{\delta} \operatorname{Pr}(\Delta \zeta<-K+s) h(s) d s, \text { and } \\
& \Delta_{3}=\frac{1}{\operatorname{Pr}\left(u_{P}-u_{\$}>\delta\right)} \int_{\delta}^{\infty} \operatorname{Pr}(\Delta \zeta<-K+s) h(s) d s .
\end{aligned}
$$


Now let $f_{1}(s):=\operatorname{Pr}(\Delta \zeta<K+s), f_{2}(s)=\operatorname{Pr}(\Delta \zeta<-K+s)$,

$$
g_{1}(s)=\left\{\begin{array}{ll}
h(s) & \text { if } s \in] \delta, \infty[, \\
0 & \text { otherwise }
\end{array} \text { and } g_{2}(s)= \begin{cases}h(s) & \text { if } s \in[0, \delta] \\
0 & \text { otherwise }\end{cases}\right.
$$

As $u_{\$}$ and $u_{P}$ are i.i.d $\operatorname{Pr}\left(0<u_{\$}-u_{P}<\delta\right)=\operatorname{Pr}\left(0<u_{P}-u_{\$}<\delta\right)$ and $\operatorname{Pr}\left(u_{\$}-u_{P}>\delta\right)=$ $\operatorname{Pr}\left(u_{P}-u_{\$}>\delta\right)$ and hence showing that $\Delta_{1} \Delta_{2}<\Delta_{0} \Delta_{3}$ boils down to showing that

$$
\int_{0}^{\infty} f_{1}(s) g_{1}(s) d s \int_{0}^{\infty} f_{2}(s) g_{2}(s) d s<\int_{0}^{\infty} f_{2}(s) g_{1}(s) d s \int_{0}^{\infty} f_{1}(s) g_{2}(s) d s .
$$

To see that this is true note that

$$
\begin{aligned}
2\left(\int_{0}^{\infty} f_{1}(s) g_{1}(s) d s \int_{0}^{\infty} f_{2}(s) g_{2}(s) d s-\int_{0}^{\infty} f_{2}(s) g_{1}(s) d s \int_{0}^{\infty}\right. & \left.f_{1}(s) g_{2}(s) d s\right) \\
& =\int_{0}^{\infty} \int_{0}^{\infty} F(x, y) G(x, y) d x d y,
\end{aligned}
$$

where $F(x, y)=f_{1}(x) f_{2}(y)-f_{1}(y) f_{2}(x)$ and $G(x, y)=g_{1}(x) g_{2}(y)-g_{1}(y) g_{2}(x)$. Further,

$$
\left(\frac{f_{1}}{f_{2}}\right)^{\prime}(s)=\frac{q(K+s) \operatorname{Pr}(\Delta \zeta<-K+s)-\operatorname{Pr}(\Delta \zeta<K+s) q(-K+s)}{(\operatorname{Pr}(\Delta \zeta<-K+s))^{2}}
$$

where $q$ is the density of $\Delta \zeta$. Then $\left(\frac{f_{1}}{f_{2}}\right)^{\prime}(s)<0$ since $0<q(K+s) \leq q(-K+s)$ and $\operatorname{Pr}(\Delta \zeta<$ $-K+s)<\operatorname{Pr}(\Delta \zeta<K+s)$ by Assumption 3. ${ }^{1}$ Thus $\frac{f_{1}}{f_{2}}$ is strictly decreasing and hence $F(x, y)>0$ if $x<y$ and $F(x, y)<0$ if $y<x$ (of course, $F(x, y)=0$ if $x=y$ ). By construction $G(x, y)>0$ if $(x, y) \in] \delta, \infty[\times[0, \delta], G(x, y)<0$ if $(x, y) \in[0, \delta] \times] \delta, \infty[$, and $G(x, y)=0$ otherwise. Hence $F(x, y) G(x, y) \leq 0$ for all $(x, y) \in \mathbb{R}_{+} \times \mathbb{R}_{+}$and $F(x, y) G(x, y)<0$ for all $(x, y) \in$ ]$\delta, \infty[\times[0, \delta] \cup[0, \delta] \times] \delta, \infty\left[\right.$. This implies that $\int_{0}^{\infty} \int_{0}^{\infty} F(x, y) G(x, y) d x d y<0$ which proves the claim.

\footnotetext{
${ }^{1}$ The fact that $q(K+s) \leq q(-K+s)$ follows by unimodality. If $s \geq K$ then $q(-K+s) \leq q(K+s)$ since $q$ is nonincreasing in the positive domain. If $s<K$ then $q(-K+s)>q(-K-s)=q(K+s)$ since $q$ is nondecreasing in the negative domain and symmetric.
} 
B Lotteries

Table 1: The lottery pairs.

\begin{tabular}{|c|c|c|c|c|c|c|c|c|}
\hline \multirow{2}{*}{$\begin{array}{c}\text { Lottery } \\
\text { pair }\end{array}$} & \multicolumn{4}{|c|}{ P-bet } & \multicolumn{4}{|c|}{$\$$-bet } \\
\hline & Prob & Outc & $\mathrm{EV}$ & StdDev & Prob & Outc & $\mathrm{EV}$ & StdDev \\
\hline 1 & 0.44 & 7 & 4.20 & 3.536 & 0.36 & 7 & 3.80 & 3.536 \\
\hline 2 & 0.40 & 8 & 4.40 & 4.243 & 0.40 & 7 & 4.00 & 3.536 \\
\hline 3 & 0.82 & 11 & 9.38 & 6.364 & 0.10 & 48 & 6.60 & 32.527 \\
\hline 4 & 0.94 & 9 & 8.58 & 4.950 & 0.20 & 30 & 7.60 & 19.799 \\
\hline 5 & 0.80 & 11 & 9.20 & 6.364 & 0.20 & 24 & 6.40 & 15.556 \\
\hline 6 & 0.90 & 10 & 9.20 & 5.657 & 0.30 & 22 & 8.00 & 14.142 \\
\hline 7 & 0.60 & 15 & 9.80 & 9.192 & 0.21 & 23 & 6.41 & 14.849 \\
\hline 8 & 0.80 & 10 & 8.40 & 5.656 & 0.40 & 15 & 7.20 & 9.192 \\
\hline 9 & 0.89 & 6 & 5.56 & 2.828 & 0.11 & 36 & 5.74 & 24.042 \\
\hline 10 & 0.81 & 6 & 5.24 & 2.828 & 0.19 & 18 & 5.04 & 11.314 \\
\hline 11 & 0.97 & 12 & 11.70 & 7.071 & 0.31 & 34 & 11.92 & 22.627 \\
\hline 12 & 0.94 & 8 & 7.64 & 4.242 & 0.39 & 16 & 7.46 & 9.899 \\
\hline 13 & 0.82 & 9 & 7.74 & 4.243 & 0.50 & 13 & 7.50 & 7.778 \\
\hline 14 & 0.87 & 7 & 6.35 & 3.536 & 0.50 & 11 & 6.50 & 6.364 \\
\hline 15 & 0.68 & 7 & 5.40 & 2.828 & 0.20 & 25 & 6.60 & 16.971 \\
\hline 16 & 0.79 & 8 & 6.74 & 2.828 & 0.30 & 24 & 8.60 & 15.556 \\
\hline 17 & 0.80 & 6 & 5.20 & 2.828 & 0.40 & 18 & 8.40 & 11.314 \\
\hline 18 & 0.90 & 6 & 5.60 & 2.828 & 0.30 & 18 & 6.80 & 11.314 \\
\hline 19 & 0.60 & 9 & 6.20 & 4.950 & 0.45 & 17 & 8.75 & 10.607 \\
\hline 20 & 0.60 & 10 & 6.80 & 5.657 & 0.40 & 16 & 7.60 & 9.899 \\
\hline
\end{tabular}

Note: Each lottery pays an amount of $€ 2$ with the remaining probability. 


\section{Screenshots}

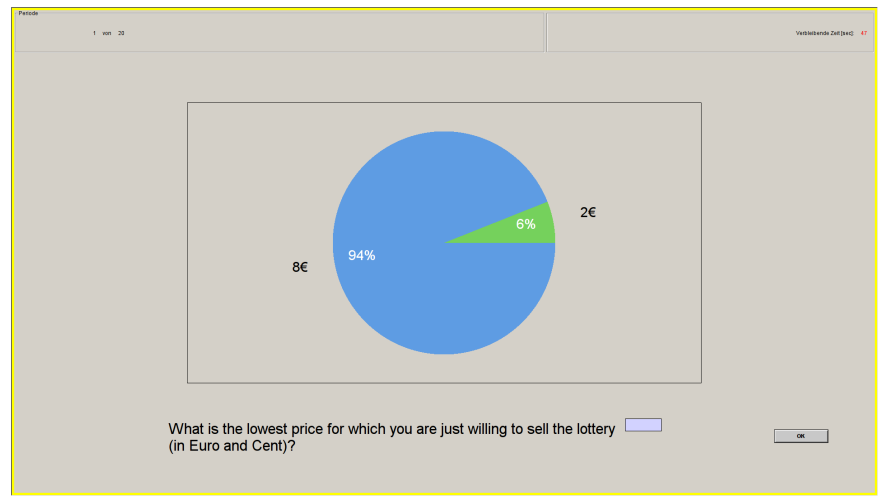

(a) Pricing phase (Experiment 1)

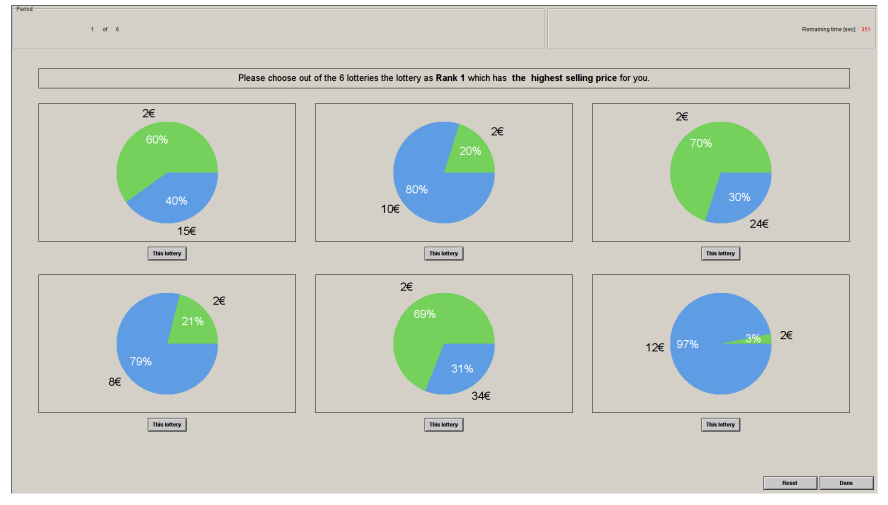

(b) Ranking phase (Experiment 2)

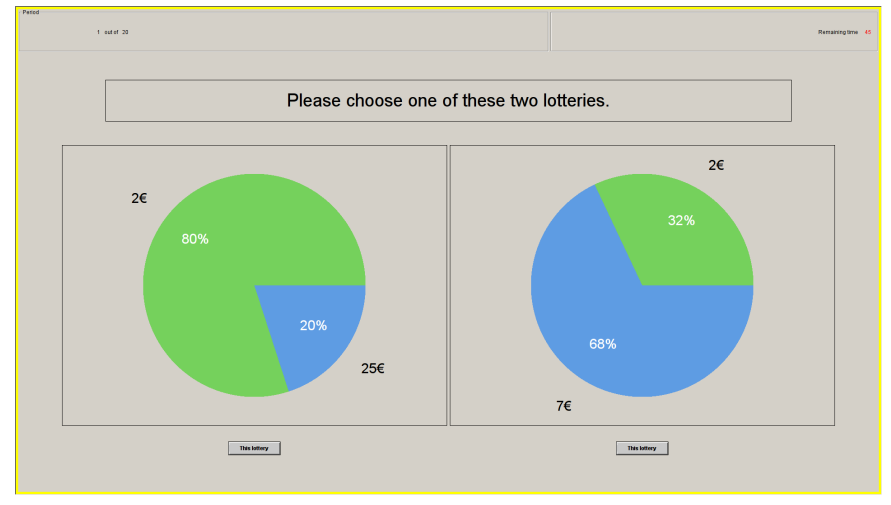

(c) Choice phase (Experiments 1 and 2)

Figure 1: Screenshots for pricing, ranking and choice task. 


\section{References}

Purkayastha, Sumitra (1998), "Simple proofs of two results on convolutions of unimodal distributions." Statistics 83 Probability Letters, 39, 97-100.

Wijsman, Robert A. (1985), "A useful inequality on ratios of integrals, with application to maximum likelihood estimation." Journal of the American Statistical Association, 80, 472475 . 